\title{
LIMITS OF FINITE DIMENSIONAL NEST ALGEBRAS
}

\author{
ALAN HOPENWASSER AND STEPHEN C. POWER
}

\begin{abstract}
We introduce order conserving embeddings as a more general form of order preserving embeddings between finite dimensional nest algebras. The structure of these embeddings is determined, in terms of order indecomposable decompositions, and they are shown to be determined up to inner conjugacy by their induced maps on $K_{0}$. Classifications of direct systems and limit algebras are obtained in terms of dimension distribution groups.
\end{abstract}

\section{CONTEnts}

1. Introduction 2

2. Regular and locally regular embeddings 6

3. Order conserving and order preserving embeddings $\quad 10$

\begin{tabular}{lll}
\hline 4. & Isomorphisms & 22
\end{tabular}

5. Invariants and classifications 31

References 40

Date: November 4, 2000.

Key words and phrases. Limit algebras, finite dimensional nest algebras, dimension distribution groups.

2000 Mathematics Subject Classification. Primary, 47L40; Secondary, 47L35.

The first author was supported by an EPSRC visiting fellowship to Lancaster University.

The second author was supported by an EPSCoR of Alabama travel grant to visit the University of Alabama. 


\section{INTRODUCTION}

One of the major themes in the study of direct systems of operator algebras is the classification of such systems and their limit algebras. Since universal results are largely out of reach and inappropriate to specific families, the usual approach is to study families of systems which are simultaneously tractable and of intrinsic interest and, for each such family, to find a complete set of invariants (up to a suitable notion of isomorphism). The selection of a family for classification entails the specification of a family of (usually finite dimensional) "building block" algebras and a class of allowable embeddings between the building block algebras. One desirable goal in the overall process is to achieve greater unification of the various classifying invariants.

The first example of this scheme was Glimm's classification of UHF $\mathrm{C}^{*}$-algebras (building blocks: full matrix algebras $\left(M_{n}(\mathbb{C})\right)$; embeddings: unital *-homomorphisms; complete invariants: the supernatural number) 88. This was eventually superseded by the Elliott's classification of $\mathrm{AF} \mathrm{C}^{*}$-algebras (building blocks: finite dimensional $\mathrm{C}^{*}$-algebras; embeddings: *-homomorphisms; complete invariants: dimension groups) [7].

Researchers in $\mathrm{C}^{*}$-algebras have proceeded on to the study of direct systems of $\mathrm{C}^{*}$-algebras which are not finite dimensional; researchers in non-self-adjoint algebras have discovered that for systems of finite dimensional (non-self-adjoint) operator algebras the limit algebras are highly dependent on both the choice of building block algebra and the choice of admissible embeddings and that the variety of limits which appear is exceptionally large. Given the central role that triangular operator algebras play in the theory of non-self-adjoint operator algebras, it is not surprising that the best understood limits in that domain are those for which the building block algebras are drawn from the family of full upper triangular matrix algebras $\left(T_{n}\right.$ 's).

Indeed, the first non-self-adjoint classification results were obtained for direct limits of $T_{n}$ 's with refinement and with standard embeddings [1, 13, 15]. This was soon followed by the classification of alternation algebras [11, 14].

These three families are subsumed by the family of direct systems in which the building blocks are $T_{n}$ 's and the embeddings are order preserving star extendible homomorphisms. Order preservation, which has its roots in [12, 17], was analysed in great detail in [3] for the $T_{n}$ context (and for direct sums thereof); in particular, a complete, albeit complicated, classification was given for order preserving limits of full upper triangular matrix algebras. 
At a greater level of generality, triangular subalgebras of $\mathrm{AF} \mathrm{C}^{*}$-algebras, with the diagonal a canonical (Cartan) masa in the $\mathrm{C}^{*}$-algebra, have played a major role in the theory of limit algebras. In particular, the spectrum (originally called the fundamental relation) serves as a complete invariant for these TAF algebras [16, 18]. A much broader class of similarly coordinatised algebras is obtained by permitting the building block algebras to be digraph algebras (including nontriangular algebras). Amongst these, a number of subclasses have proven to be classifiable in terms of scaled abelian groups. A notable example is the family of $2 n$-cycle algebras, which are of particular interest since these are the simplest operator algebras with non-trivial homology. With rigid star extendible embeddings, these have been classified in [6, 5, 19].

Like triangular algebras, nest algebras have played a critical role, since its inception, in the theory of non-self-adjoint algebras. This paper is devoted to a study of direct systems in which the building blocks are finite dimensional nest algebras. For invariants, we turn to the Grothendieck dimension distribution groups, which were first developed in [20]. These will be denoted by $\mathcal{G}$. This invariant reduces to $K_{0}$ in the $\mathrm{C}^{*}$-algebra context and to a dual form of the spectrum in the TAF algebra context. Dimension distribution groups, augmented as necessary by various ancillary structures, form an effective invariant for additional families of systems - as we shall see with finite dimensional nest algebras - and help unify the classification of direct systems and of limit algebras.

It is, however, necessary to restrict the class of admissible embeddings between finite dimensional nest algebras in order to obtain classification theorems in these terms. We shall show that concepts of order preservation and conservation give rise to diverse embeddings which are tractable in this way. Order preservation for embeddings is defined in terms of order preservation for isometries; in [2], Donsig extended these concepts from the $T_{n}$ context to the digraph algebra context. The definitions are made with respect to a self-adjoint subalgebra of the original algebra; in the triangular case the diagonal is the only reasonable choice for this subalgebra. When $A$ is a finite dimensional nest algebra, $A \cap A^{*}$ is a natural choice for this subalgebra; we adopt Donsig's definition in the context of this paper.

We shall find, however, that critical preliminary theorems $\left(K_{0}\right.$-uniqueness and $\mathcal{G}$-lifting) are valid for broader classes of embeddings and so classification can be achieved beyond the realm of order preserving systems. A related concept, order conservation, is central for this extension. The reason this new concept appears for finite dimensional nest algebras, 
and did not appear for $T_{n}$ 's, is that the diagonal order on projections is not anti-symmetric in the former context as it is in the latter. With the understanding that we consider only regular partial isometries (see section 2), we define an order preserving partial isometry to be one for which conjugation by the partial isometry preserves the diagonal order ( $p \preceq q$ if, and only if, $v p v^{*} \preceq v q v^{*}$, where $p$ and $q$ are subprojections of the initial space of the normalizing partial isometry $v$ ). We define order conserving partial isometries to be ones which respect, but do not necessarily preserve, the diagonal order (in the sense that $p \prec q$ and $v q v^{*} \prec v p v^{*}$ cannot simultaneously hold). In the $T_{n}$ context, these two concepts coincide. But for finite dimensional nest algebras in which at least some of the atoms have rank greater than one, the order preserving partial isometries form a proper subset of the order conserving partial isometries.

Order preserving embeddings are defined to be those embeddings which map order preserving partial isometries to order preserving partial isometries. Similarly, order conserving embeddings map order conserving partial isometries to order conserving partial isometries. In the $T_{n}$ context, these two families of embeddings coincide. In general, however, neither family contains the other. Examples illustrating these facts are given at the end of Section 3 .

There are local variants of these concepts which are of importance. An embedding is locally order preserving if it maps rank one (regular) partial isometries to order preserving partial isometries and is locally order conserving if it maps rank one (regular) partial isometries to order conserving partial isometries. Clearly, each property implies its local variant; furthermore, local order preservation implies local order conservation (since order preserving partial isometries are order conserving). The following summarizes these relationships:

$$
\begin{aligned}
& O P \Longrightarrow L O P \Longrightarrow L O C \\
& O C \Longrightarrow L O C
\end{aligned}
$$

Thus, the locally order conserving embeddings form the broadest family, containing all the other families. Happily, two properties essential for dimension distribution group classification, $K_{0}$-uniqueness and $\mathcal{G}_{\text {- }}$ lifting, are valid for all locally order conserving embeddings. See Theorem 3.2 and Theorem 5.2. These relationships and facts indicate the importance of a purview broader than order preservation.

Based on these results, we prove that the dimension distribution group, together with ancillary structures, is a complete invariant for systems of finite dimensional nest algebras of four types, one for each preservation/conservation property. The classification is up to regular 
isomorphism of the appropriate preservation/conservation type. See Theorems 5.6, 5.7, 5.8, and 5.9.

The classification of operator algebra direct limits involves substantial technical difficulties and has been deferred. However, for algebraic direct limits (locally finite algebras), we obtain two classification theorems - one for limits of order preserving systems using the dimension distribution group and an order preserving scale and one for limits of order conserving systems using the dimension distribution group and an order conserving scale. See Theorems 5.10 and 5.11 .

We now briefly indicate related classification schemes for nontriangular limit algebras. For nest algebras with just two atoms, star extendible embeddings are automatically order conserving (although not necessarily order preserving). The corresponding $T_{2}$-limit algebras $A$ were classified in [17] and in [10] in terms of an augmentation of $K_{0}$ data by a binary relation on $\Sigma_{0}(A) \times \Sigma_{0}(A)$ where $\Sigma_{0}(A)$ is the $K_{0}$ scale of $A$. Also, Donsig [2] has obtained a complete classification of order preserving limits of general finite dimensional nest algebras (and more general chordal algebras) by means of a ternary relation augmentation of $K_{0}$ data. On the other hand, away from order conservation, in [21] $T_{r}$-limit algebras, with general star extendible embeddings, are classified in terms of metrized dimension module invariants.

Here is a guide to the four remaining sections of this paper. Section 2 discusses regular embeddings between finite dimensional nest algebras. In particular, we show that local regularity implies regularity in the finite dimensional nest algebra context. This result is not valid for general digraph algebras.

Section 3 introduces the various concepts of order preservation and order conservation. The $K_{0}$-uniqueness theorem for locally order conserving embeddings is proven. This is a stronger result than we actually need for classification; $\mathcal{G}$-uniqueness would suffice. This section also contains a digression (which the reader interested solely in classification may skip) into the structure of order conserving embeddings. We obtain a result (Corollary 3.10) analogous to the theorem in [3] which states that an order preserving embedding in the $T_{n}$ context is an ordered sum of refinement embeddings. For this purpose, we need to introduce refinement type embeddings (a generalization of refinement embeddings) and $T_{2}$-degenerate embeddings, which are trivially order conserving but may not be of refinement type. Curiously, in order for the theorem to be valid, we must assume that every atom of the domain algebra has rank two or greater. Thus, a structure therem for order conserving embeddings is available when all atoms have rank one or when all atoms have rank greater than one. For the case with 
mixed rank one and multi-rank atoms, we show by counterexample that the theorem is false. (A study of examples suggests that some sort of general structure theorem is valid, but that the basic order irreducible types are sufficiently complicated and special that such a theorem may be of dubious interest.)

Section 4 studies isomorphism of the various systems of interest. To obtain classifications of the algebraic limits up to star extendible isomorphism we require the result that a general star extendible isomorphism between the limit algebras of locally order conserving systems necessarily derives from a regular isomorphism between the given systems (Theorem 4.3). This guarantees that our invriants for limit algebras are well-defined. Moreover, we refine this result (in Theorem 4.4) to enable the formulation of invariants defined in terms of order preserving or order conserving maps (rather than regular maps).

Section 5 gives a brief introduction to partial isometry dimension distribution groups. For more detail, the reader is referred to [20]. The $\mathcal{G}$-lifting theorem for locally order conserving embeddings is proven (and yields $\mathcal{G}$-lifting for all the other types of embeddings) and various scales are defined for dimension distribution groups. The six classification theorems described earlier in the introduction are proven and the section concludes with several examples. Readers may wish to glance at these examples before perusing the whole paper.

The authors thank Allan Donsig and Paul Haworth for several helpful comments.

\section{REgUlAR AND LOCALLY REGULAR EMBEDDINGS}

A finite dimensional nest algebra is a unital operator algebra $A$ in $M_{n}=M_{n}(\mathbb{C})$ which has a block upper triangular form with respect to an ordered decomposition of the identity as an orthogonal sum of projections: $1=q_{1}+q_{2}+\ldots+q_{l}$. Such an algebra $A$ consists of all those operators $a$ in $M_{n}$ for which $(1-f) a f=0$ for all projections $f=q_{1}+\ldots+q_{k}, 1 \leq k \leq l$. The projections $f$, together with the zero projection, form the invariant projection nest for $A$, while the projections $q_{i}$ are the atomic interval projections of $\mathrm{A}$.

The nest algebra $A$ induces, through its atomic interval projections, a block structure on $M_{n}$ whereby each matrix $v$ in $M_{n}$ can be viewed as an $l \times l$ block matrix $v=\left(v_{i j}\right)$ where $v_{i j}=q_{i} v q_{j}$. The elements of the nest algebra are precisely the block upper triangular matrices with respect to this block structure. Unless some other block structure is explicitly specified, it will always be assumed that elements of a nest algebra have the block structure natural to the nest. 
Definition 2.1. A partial isometry $v$ in $M_{n}$ is said to be regular with respect to a block structure if each block matrix entry is a partial isometry.

Note that any two block matrix entries in the same row of a regular partial isometry have orthogonal final spaces and any two block matrices in the same column have orthogonal initial spaces.

Definition 2.2. A partial isometry $v$ in a finite dimensional $\mathrm{C}^{*}$-algebra is said to be standard, with respect to a matrix unit system if it is a sum of some of these matrix units.

Note that if a partial isometry is standard with respect to a matrix unit system, then it is regular with respect to any block structure compatible with that matrix unit system.

An algebra homomorphism $\phi: A_{1} \rightarrow A_{2}$ between two nonself-adjoint operator algebras is said to be star extendible if it is the restriction of a star algebra homomorphism $\tilde{\phi}$ between the generated $\mathrm{C}^{*}$-algebras. Plainly, $\tilde{\phi}$ is uniquely determined by $\phi$. In the next definition and throughout the paper we confine attention to such star-extendible homomorphisms.

Definition 2.3. Let $\phi: A_{1} \rightarrow A_{2}$ be a star-extendible homomorphism between finite dimensional nest algebras. Then $\phi$ is said to be regular if $\phi$ is a direct sum of multiplicity one embeddings. Also, $\phi$ is locally regular if $\phi(v)$ is a regular partial isometry in $A_{2}$ whenever $v$ is a regular partial isometry in $A_{1}$.

Remarks. If $\phi$ is a regular embedding then $\phi$ is locally regular. We prove the converse in Theorem 2.4 below. Although the definition above makes sense for maps between digraph algebras it is not generally true that local regularity is equivalent to regularity. For example, consider the algebra $E \subseteq T_{3}$ which is spanned by all the matrix units of $T_{3}$ except $e_{23}$. Then one can verify that every star extendible map $\phi: E \rightarrow E \otimes M_{n}$ for $n \geq 2$ is locally regular, and yet there are irregular (i.e. nonregular) embeddings. See also the 4-cycle algebra example, Example 2.2, in [6].

In order to show that a star-extendible map $\phi$ is locally regular, it is sufficient to show that $\phi(v)$ is a regular partial isometry in $A_{2}$ whenever $v$ is a rank-one regular partial isometry in $A_{1}$. To see this, let $\tilde{Q}_{1}, \ldots, \tilde{Q}_{m}$ be the atomic interval projections of $A_{1}$ and $Q_{1}, \ldots, Q_{n}$ the atomic interval projections of $A_{2}$. Let $v$ be a regular partial isometry in $A_{1}$. Then $v_{i j}=\tilde{Q}_{i} v \tilde{Q}_{j}$ is a partial isometry, for all $i$ and $j$. Each $v_{i j}$, in turn, can be written a sum of rank one partial isometries each 
of which has the form $w=\tilde{Q}_{i} w \tilde{Q}_{j}$. Thus, we may write $v$ as a sum, $v_{1}+\cdots+v_{p}$ say, of rank one regular partial isometries

Since $\phi$ is star extendible, $\phi(v)=\phi\left(v_{1}\right)+\cdots+\phi\left(v_{p}\right)$ is a partial isometry and, by hypothesis, each $\phi\left(v_{i}\right)$ is a regular partial isometry in $A_{2}$. From this we may conclude that the set of initial projections of the $\phi\left(v_{i}\right)$ are pairwise orthogonal, the set of final projections of the $\phi\left(v_{i}\right)$ are pairwise orthogonal, and both the initial projection and the final projection of each $\phi\left(v_{i}\right)$ commute with each atom $Q_{t}$. The last statement follows from the fact that every $Q_{s} \phi\left(v_{i}\right) Q_{t}$ is a partial isometry.

It now follows that, for all $s$ and $t$, the set of initial projections of the elements $Q_{s} \phi\left(v_{i}\right) Q_{t}(i=1, \ldots p)$ are pairwise orthogonal; the same is true for the set of final projections. Consequently, $Q_{s} \phi(v) Q_{t}=$ $Q_{s} \phi\left(v_{1}\right) Q_{t}+\cdots+Q_{s} \phi\left(v_{p}\right) Q_{t}$ is a partial isometry. Since this is true for all $s$ and $t$, we see that $\phi(v)$ is regular in $A_{2}$ and that $\phi$ is locally regular.

We may, in fact, go a bit further than reducing local regularity to the action of $\phi$ on rank-one regular partial isometries. If $\left\{e_{i j}\right\}$ is a matrix unit system in $A_{1}$ compatible with the block matrix structure of $A_{1}$ and if $\phi\left(e_{i j}\right)$ is regular in $A_{2}$ for all $e_{i j}$, then $\phi$ is locally regular.

If $\phi$ is locally regular and $\left\{e_{i j}\right\}$ is a matrix unit system compatible with $A_{1}$, then for each matrix unit $e_{i j}$ there is a matrix unit system for $A_{2}$ with respect to which $\phi\left(e_{i j}\right)$ is a sum of matrix units; i.e. $\phi\left(e_{i j}\right)$ is standard. If, moreover, there is a single matrix unit system for $A_{2}$ with respect to which all $\phi\left(e_{i j}\right)$ are standard, then it follows that $\phi$ is regular. Obtaining such a system will be the strategy for the proof of the following theorem.

Theorem 2.4. Let $\phi: A_{1} \rightarrow A_{2}$ be a star extendible homomorphism between finite dimensional nest algebras. If $\phi$ is locally regular then $\phi$ is regular.

Proof. The nest algebra $A_{2}$ and the $\mathrm{C}^{*}$-algebra it generates have an $r \times r$ block structure induced by the $r$ atoms in the nest for $A_{2}$. In this block structure, the diagonal blocks are square matrices of varying size and the off-diagonal blocks are rectangular. Elements of $A_{2}$ and $C^{*}\left(A_{2}\right)$ will be written as $r \times r$ matrices, $v=\left(v_{s t}\right), 1 \leq s, t \leq r$, where each $v_{s t}$ is a matrix of appropriate size.

We may assume that $A_{1}=T_{n}$. Consider the star algebra extension of $\phi$. This is an algebra injection $\phi: M_{n} \rightarrow C^{*}\left(A_{2}\right)$ such that the image of each standard matrix unit of $M_{n}$ is a regular partial isometry. We wish to show that $\phi$ is regular with respect to the $r$ by $r$ block structure. 
By the remarks above this is equivalent to showing the following with $v^{i j}=\phi\left(e_{i j}\right)$.

Let $v^{i j}=\left(v_{s t}^{i j}\right)$,with $1 \leq s, t \leq r, 1 \leq i, j \leq n$, be a matrix unit system for a subalgebra of $C^{*}\left(A_{2}\right)$ which is isomorphic to $M_{n}$. Suppose moreover that each $v^{i j}$ is regular with respect to the $r$ by $r$ block structure, so that each block matrix entry $v_{s t}^{i j}$ of each $v^{i j}$ is itself a partial isometry. We show that there is a matrix unit system for $C^{*}\left(A_{2}\right)$, which consists of (rank one) regular partial isometries, such that each $v^{i j}$ is a sum of some of these matrix units.

To see this consider first a product $v^{i j} v^{j k}$. The 1,1 block entry is given by the sum

$$
v_{11}^{i j} v_{11}^{j k}+v_{12}^{i j} v_{21}^{j k}+\cdots+v_{1 r}^{i j} v_{r 1}^{j k} .
$$

Since $v^{i j}$ is regular, the partial isometries $v_{11}^{i j}, \ldots, v_{1 r}^{i j}$ have orthogonal range projections and so the operators of the sum have orthogonal range projections. For similar reasons the domain projections are pairwise orthogonal. (We are not assuming here that these products are partial isometries.) Since, by hypothesis, the product $v^{i j} v^{j k}$ is a regular partial isometry, it follows that the sum above is a partial isometry, and therefore, by the orthogonality of domain and range projections, each of the individual products

$$
v_{11}^{i j} v_{11}^{j k}, v_{12}^{i j} v_{21}^{j k}, \ldots, v_{1 r}^{i j} v_{r 1}^{j k}
$$

is a partial isometry.

Now, since, for example, $v_{11}^{i j} v_{11}^{j k}$ is a partial isometry it follows that the range projection of $v_{11}^{j k}$ commutes with the domain projection of $v_{11}^{i j}$. Abusing notation somewhat, and regarding the entry operators $v_{s t}^{i j}$ as identified with operators in $C^{*}\left(A_{2}\right)$, it follows, by considering other block entries, that for all $i, j, k, l, s, t, u, v$ the range projection of $v_{s t}^{i j}$ commutes with the domain projection of $v_{u v}^{k l}$. Note also that the domain projections and the range projections commute amongst themselves. Furthermore it is clear that these projections commute with the projections in the centre of the block diagonal subalgebra of $C^{*}\left(A_{2}\right)$. Choose now a maximal family of rank one projections $p$ which commute with all these projections and are dominated by $v_{11}^{*} v_{11}$. Then for each such projection the set of operators $w_{i j}=v^{i 1} p v^{1 j}$ satisfy the relations of a matrix unit system. The projections $P=\Sigma_{i} v^{i 1} p v^{1 i}$ are pairwise orthogonal and decompose $\phi$ as a direct sum of multiplicity one embeddings, as desired. 


\section{ORDER CONSERVING AND ORDER PRESERVING EMBEDDINGS}

In this section we define order conserving and locally order conserving star extendible embeddings of finite dimensional nest algebras. We show that these embeddings are classified up to inner conjugacy by $K_{0}$ and we make use of this in the classifications of Section 5. This inner conjugacy classification can also be viewed as a classification of those subalgebras $A_{1}$ of a fixed nest algebra $A_{2}$ where $A_{1}$ is a nest algebra in $C^{*}\left(A_{1}\right)$ and where the inclusion of $A_{1}$ is an order conserving inclusion.

In [3], it is shown that unital order preserving embeddings in the $T_{n}$ context are direct sums of refinement embeddings. We will extend this result (slightly) to non-unital embeddings and will obtain an analogous decomposition for order conserving embeddings between nest algebras, provided that all atoms in the domain algebra have rank greater than one. The decomposition of $\phi$ will be into an ordered sum of order irreducible embeddings of two basic types to be defined below.

In this paper we consider a general finite dimensional operator algebra $A$ to be a nest algebra if it is star extendibly isomorphic to a nest algebra. This means that the generated $\mathrm{C}^{*}$--algebra $C^{*}(A)$ is isomorphic to $M_{p}$ for some $p$ and $A$ is a nest algebra in $C^{*}(A)$.

Definition 3.1. Let $A \subseteq M_{n}$ be a finite dimensional nest algebra with atomic interval projections $q_{1}, q_{2}, \ldots, q_{l}$.

1. The set $\operatorname{Pisom}_{\text {reg }}(A)$ is the set of regular partial isometries with respect to the block structure from $q_{1}, \ldots, q_{l}$.

2. The set $\operatorname{Pisom}_{\mathrm{oc}}(A)$ denotes the subset of $\mathrm{Pisom}_{\mathrm{reg}}(A)$ consisting of order conserving partial isometries $v$; that is, those for which

$$
q_{i} v q_{j} \neq 0 \Longrightarrow q_{s} v q_{t}=0, \text { for } s<i \text { and } t>j .
$$

3. A star extendible embedding between finite dimensional nest algebras is order conserving if it maps order conserving partial isometries to order conserving partial isometries.

4. An embedding $\phi: A_{1} \rightarrow A_{2}$ is locally order conserving if $\phi(v) \in$ $\operatorname{Pisom}_{\mathrm{oc}}\left(A_{2}\right)$ for each rank one element $v$ in $\operatorname{Pisom}_{\text {reg }}\left(A_{1}\right)$.

If $v \in \operatorname{Pisom}_{\text {reg }}(A)$ and $\operatorname{rank} v=1$ then $v \in \operatorname{Pisom}_{\mathrm{oc}}(A)$. It follows from this observation, the remarks following Definition 2.3, and Theorem 2.4 that a locally order conserving embedding between finite dimensional nest algebras is automatically a regular embedding.

In particular, order conserving star extendible embeddings are regular star extendible embeddings and so admit decompositions as direct sums of multiplicity one embeddings. 
For an explicit example of an order conserving embedding consider the map $\phi$ from $T(2,2,2)$ to $T(6,8,10)$ given by

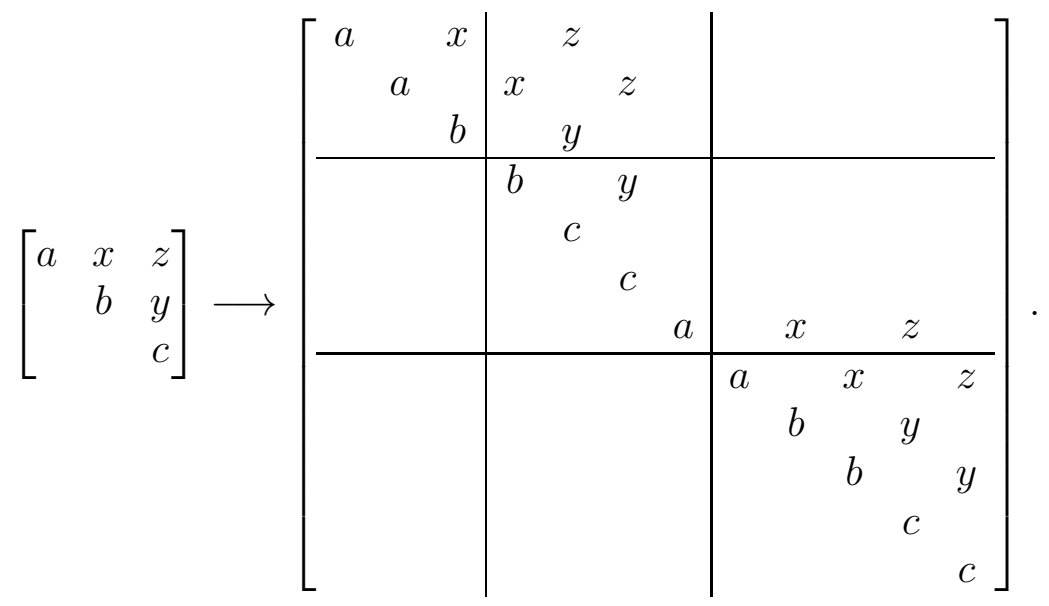

This map has an order irreducible ordered sum decomposition $\phi_{1}+$ $\phi_{2}+\phi_{3}$ where $\phi_{1}$ is a multiplicity 2 map, with range dominated by the sum of the first two atoms of the codomain algebra, and $\phi_{2}$ and $\phi_{3}$ are multiplicity one embeddings. The summands here are all of $T_{2}$-degenerate type (in the sense below).

In general, the order conserving partial isometries in $A$ are those regular partial isometries whose block matrix supports have a staircase form, such as

$$
\left[\begin{array}{cccccc}
* & * & * & 0 & 0 & 0 \\
& 0 & * & 0 & 0 & 0 \\
& & * & * & * & 0 \\
& & & 0 & * & 0 \\
& & & & * & * \\
& & & & & *
\end{array}\right] .
$$

Theorem 3.2. Let $A_{1}$ be a finite dimensional nest algebra. Let $\phi$ and $\psi$ be two locally order conserving regular embeddings of $A_{1}$ into a second finite dimensional nest algebra, $A_{2}$. Then $\phi$ and $\psi$ are inner conjugate if, and only if, $K_{0}(\phi)=K_{0}(\psi)$.

Proof. We first prove the theorem for the special case in which every atom of $A_{1}$ has rank one (i.e. $A_{1}$ is some $T_{n}$ ). Select a matrix unit system for $C^{*}\left(A_{1}\right)$ compatible with $A_{1}$; for each pair of (rank one) atoms $s$ and $r$, let $m(s, r)$ denote the matrix unit with initial projection $r$ and final projection $s$. Note that $K_{0}(\phi)$ is a matrix whose columns are indexed by the atoms of $A_{1}$ and whose rows are indexed by the atoms of $A_{2}$. The entry in the column indexed by the atom $r$ of $A_{1}$ and the row indexed by the atom $Q$ of $A_{2}$ is the non-negative integer rank $\phi(r) Q$. 
We assume the order of the rows and columns of this matrix reflect the usual $\prec$ ordering of the atoms of $A_{1}$ and $A_{2}$.

Let $r$ be an atom of $A_{1}$ with the property that the first non-zero entry in the column of $K_{0}(\phi)$ indexed by $r$ is less than or equal to the first non-zero entry in any other column. Let $Q$ be the atom which indexes the row containing the first non zero entry in column $r$. Now assume that $s$ is some other atom of $A_{1}$. First, assume $s \prec r$. Let $P$ be the atom of $A_{2}$ which gives the first non-zero entry in column $s$. Let $E_{r}=\phi(r) Q$ and $F_{s}=\phi(s) P$. The fact that $\phi$ is locally order conserving ensures that $\phi(m(s, r)) E_{r} \phi(m(s, r))^{*} \leq F_{s}$. To see this note that for an appropriate choice of matrix units in $A_{2}, \phi(m(s, r))$ induces an order conserving bijection from the rank one matrix unit subprojections of $\phi(r)$ to the rank one matrix unit subprojections of $\phi(s)$. Since $E_{r}$ and $F_{s}$ correspond to the first block occurences of these matrix unit projections and since, by the choice of $r, F_{s}$ has at least as many of them as $E_{r}$ does, it follows that $\phi(m(s, r))$ conjugates $E_{r}$ to a subprojection of $F_{s}$.

Let $E_{s}=\phi(m(s, r)) E_{r} \phi(m(s, r))^{*}$. If $r \prec s$, we argue in a similar fashion to obtain a projection $E_{s}$ which is a subprojection of the index atom for the row containing the first non-zero entry in column $s$ and which satisfies $E_{r}=\phi(m(r, s)) E_{s} \phi(m(r, s))^{*}$. The fact that $\phi$ is an embedding implies that, for any two atoms $s \prec s^{\prime}, E_{s}=$ $\phi\left(m\left(s, s^{\prime}\right) E_{s^{\prime}} \phi\left(m\left(s, s^{\prime}\right)\right)^{*}\right.$.

Note that if $s$ and $s^{\prime}$ are distinct atoms of $A_{1}$, then the projections $E_{s}$ and $E_{s^{\prime}}$ are orthogonal. This is true even if $E_{s}$ and $E_{s^{\prime}}$ are subprojections of the same atom of $A_{2}$, since $E_{s} \leq \phi(s), E_{s^{\prime}} \leq \phi\left(s^{\prime}\right)$ and $\phi(s)$ and $\phi\left(s^{\prime}\right)$ are orthogonal projections.

Let $P_{1}=\sum_{s} E_{s}$, where the sum runs over all atoms of $A_{1}$. Let $\phi_{1}=P_{1} \phi P_{1}$. It follows from the way that $\phi_{1}$ is defined that $\phi_{1}$ and $\left(1-P_{1}\right) \phi\left(1-P_{1}\right)$ are locally order conserving embeddings of $A_{1}$ into $A_{2}$ and that $\phi=\phi_{1}+\left(1-P_{1}\right) \phi\left(1-P_{1}\right)$. The reason that $\phi_{1}$ and $\left(1-P_{1}\right) \phi\left(1-P_{1}\right)$ are locally order conserving is that any subpartial isometry of an order conserving partial isometry which is obtained by left and right multiplication by a block diagonal projection is again order conserving. Note that $K_{0}\left(\left(1-P_{1}\right) \phi\left(1-P_{1}\right)\right)$ is obtained from $K_{0}(\phi)$ by subtracting from the first non-zero entry in each column of $K_{0}(\phi)$ the integer in column $r$, row $Q$.

Since $K_{0}(\psi)=K_{0}(\phi)$, we may construct a projection $P_{1}^{\psi}$ which has the same properties with respect to $\psi$ that $P_{1}$ has with respect to $\phi$. $P_{1}^{\psi}$ is a sum of projections, one for each atom of $A_{1}$; the projection in the sum associated with an atom $s$ has the same rank as $E_{s}$ and is a subprojection of the same atom of $A_{2}$ as $E_{s}$. Consequently, there is a 
unitary element of the diagonal $A_{2} \cap A_{2}^{*}$ such that if $\tilde{\psi}=u^{*} \psi u$, then $P_{1} \phi P_{1}=P_{1} \tilde{\psi} P_{1}$ and $\left(1-P_{1}\right) \tilde{\psi}\left(1-P_{1}\right)$ is an embedding.

We may now repeat this procedure with the two embeddings, $(1-$ $\left.P_{1}\right) \phi\left(1-P_{1}\right)$ and $\left(1-P_{1}\right) \tilde{\psi}\left(1-P_{1}\right)$. When we do so, we obtain a projection $P_{2}$ which is orthogonal to $P_{1}$ and an embedding $\hat{\psi}$ which is inner conjugate to $\psi$ such that $P_{2} \phi P_{2}=P_{2} \hat{\psi} P_{2}$. Furthermore, because we are really working in essence on $1-P_{1}$, we can select the unitary which implements the conjugacy so that it is the identity on $P_{1}$. With this done, we also have $P_{1} \phi P_{1}=P_{1} \hat{\psi} P_{1}$ and hence $\left(P_{1}+P_{2}\right) \phi\left(P_{1}+P_{2}\right)=$ $\left(P_{1}+P_{2}\right) \hat{\psi}\left(P_{1}+P_{2}\right)$. It is now clear that if we continue in this fashion, after finitely many steps we obtain an inner conjugacy between $\phi$ and $\psi$.

Slight modifications of the argument given above yield the proposition when $A_{1}$ is a general finite dimensional nest algebra. Alternatively, we can deduce the general version of the proposition from the special case above by the following 'general principles' technique. If $A_{1}$ is a general nest algebra, select a subalgebra $B$ of $A_{1}$ which is isomorphic to some $T_{n}$ such that each block of $A_{1} \cap A_{1}^{*}$ corresponding to an atom of $A_{1}$ contains exactly one rank one diagonal projection from $B$. Note that $A_{1}$ is the algebra generated by $B$ and $A_{1} \cap A_{1}^{*}$. Let $\phi$ and $\psi$ be two locally order conserving embeddings such that $K_{0}(\phi)=K_{0}(\psi)$. Since $\phi$ and $\psi$ are star extendible, they are determined (up to inner equivalence) by their restrictions to $B$. But $K_{0}\left(\left.\phi\right|_{B}\right)=K_{0}(\phi)=K_{0}(\psi)=K_{0}\left(\left.\psi\right|_{B}\right)$. By the argument above, $\left.\phi\right|_{B}$ and $\left.\psi\right|_{B}$ are inner equivalent; it follows immediately that $\phi$ and $\psi$ are inner equivalent.

3.1. Structure theorems. We now determine the way in which order conserving embeddings decompose as ordered sums of embeddings of special types. Our original motivation for studying such decompositions was to use these as a vehicle for obtaining $K_{0}$-uniqueness. Theorem 3.2, however, provides $K_{0}$-uniqueness more directly and for a broader class of embeddings. While the original motivation is no longer germane, the structure theorem is of independent interest. We will also comment on connections with $K_{0}$-uniqueness, since these connections are illuminating.

In the next definition we write $\prec_{A}$ to indicate the total ordering on the atomic projections of a nest algebra $A$.

Definition 3.3. (i) A (possibly nonunital) star extendible embedding $\phi: T_{n} \rightarrow T_{m}$ is said to be a refinement type embedding if $\phi$ is locally order conserving and if, whenever $e_{1} \prec_{T_{n}} e_{2}$ and $e_{1} \neq e_{2}$, it follows that $f_{1} \prec_{T_{m}} f_{2}$ for $f_{1} \leq \phi\left(e_{1}\right)$ and $f_{2} \leq \phi\left(e_{2}\right)$. 
(ii) A (possibly nonunital) star extendible embedding $\phi: A_{1} \rightarrow A_{2}$ between finite dimensional nest algebras is said to be of refinement type if there are maximal triangular subalgebras $T_{n} \subseteq A_{1}, T_{m} \subseteq A_{2}$ such that $\phi\left(T_{n}\right) \subseteq T_{m}$ and the restriction map $\phi: T_{n} \rightarrow T_{m}$ is of refinement type.

A refinement type embedding is necessarily order conserving. To see this let $\rho: T_{n} \rightarrow T_{m}$ be a refinement embedding with star extension $\rho: M_{n} \rightarrow M_{m}$ and let $A_{1}$ be a nest algebra containing $T_{n}$. The algebra $A_{1}$ necessarily has a standard block upper triangular structure. There is a smallest nest algebra $A_{2}$ containing $T_{m}$ and $\rho\left(A_{1}\right)$ and this algebra also has standard form. Note that the images of the atoms of rank greater than one are atoms of $A_{2}$ whilst the other atoms of $A_{2}$ are of rank one. The map $\rho: A_{1} \rightarrow A_{2}$ is clearly order conserving. If $A_{3}$ is a nest algebra in $M_{m}$ containing $A_{2}$ then since the block structure of $A_{2}$ refines that of $A_{3}$ it follows that $\operatorname{Pisom}_{\mathrm{oc}}\left(A_{2}\right) \subseteq \operatorname{Pisom}_{\mathrm{oc}}\left(A_{3}\right)$. Thus $\rho: A_{1} \rightarrow A_{3}$ is order conserving and such a map $\rho$ is a typical unital refinement type embedding.

A general refinement type embedding $\phi: A_{1} \rightarrow A_{4}$ has the form $\psi \circ \rho$ where $\psi: A_{3} \rightarrow A_{4}$ is a multiplicity one inclusion, and so is also order conserving.

Note that the argument above implies that the image of an atom of rank greater than one, under a refinement type embedding, is dominated by an atom of the range.

Definition 3.4. Let $\phi_{i}: A_{1} \rightarrow A_{2}, 1 \leq i \leq r$, be star extendible algebra homomorphisms between finite dimensional nest algebras $A_{1}, A_{2}$ such that the projections $\phi_{i}(1)$ are pairwise orthogonal. Then the star extendible embedding $\phi_{1}+\cdots+\phi_{r}$ is said to be an ordered sum if

$$
q \phi_{i}(1) \neq 0 \Longrightarrow q^{\prime} \phi_{j}(1)=0 \quad \text { for } \quad q \prec q^{\prime}, j<i
$$

for all atomic interval projections $q, q^{\prime}$ of $A_{2}$, and for all $i$.

What this means can also be described in terms of a choice of a maximal set $\mathcal{P}=\left\{e_{1}, e_{2}, \ldots, e_{m}\right\}$ of rank one projections in $A_{2}$ whose indexing is consistent with the partial ordering $\prec_{A_{2}}$ on the set $\mathcal{Q}$ of atomic interval projections $q$ (in the sense that if $e_{i} \leq q, e_{j} \leq q^{\prime}, i \leq j$, then $\left.q \preceq_{A_{2}} \quad q^{\prime}\right)$ and is such that each projection $\phi_{i}(1)$ is a sum of some of the projections of $\mathcal{P}$. With any such choice of $\mathcal{P}$ the condition of the last definition holds if the $\mathcal{P}$-support of the projections $\phi_{1}(1), \phi_{2}(1), \ldots, \phi_{r}(1)$ appear in order, that is,

$$
\max \left\{j: e_{j} \phi_{i}(1) \neq 0\right\}<\min \left\{j: e_{j} \phi_{i+1}(1) \neq 0\right\}
$$

for each $i=1, \ldots, r$. 
Definition 3.5. Let $\phi: A_{1} \rightarrow A_{2}$ be a star extendible algebra homomorphism with ordered decomposition $\phi_{1}+\cdots+\phi_{r}$. Such a decomposition is said to be order reducible if for some index $i$ the map $\phi_{i}$ has a non-trivial ordered sum decomposition.

The following proposition is evident.

Proposition 3.6. A star extendible embedding between finite dimensional nest algebras admits an order irreducible ordered sum decomposition which is unique up to a permutation of multiplicity one summands which map into the same diagonal block. Moreover the embedding is order conserving if, and only if, each summand is an order conserving embedding.

This proposition provides a useful perspective and to illustrate this we prove a key result from [3] generalised to the case of nonunital maps.

Proposition 3.7. Let $n \geq 3$ and let $\phi: T_{n} \rightarrow T_{m}$ be a star extendible embedding. Then $\phi$ is an order conserving embedding if, and only if, $\phi$ is an ordered sum $\phi=\phi_{1}+\cdots+\phi_{p}$, where each $\phi_{j}$ is a refinement type embedding.

Proof. Let $\phi$ be order conserving with order-irreducible ordered decomposition

$$
\phi_{1}+\phi_{2}+\cdots+\phi_{l}
$$

and let $\mathcal{P}=\left\{e_{1}, \ldots, e_{n}\right\}$ be the atomic interval projections of $T_{n}$, listed in their natural order. Let $Q_{1}, Q_{2}, \ldots, Q_{j}$ be consecutive interval projections of $T_{m}$ (consecutive in the sense of there being no gaps) chosen with the following properties:

$Q_{i} \phi\left(e_{i}\right)=Q_{i} \phi(1)$, for each $i$, $\operatorname{rank}\left(Q_{1} \phi\left(e_{1}\right)\right)=\operatorname{rank}\left(Q_{2} \phi\left(e_{2}\right)\right)=\cdots=\operatorname{rank}\left(Q_{j-1} \phi\left(e_{j-1}\right)\right) \geq \operatorname{rank}\left(Q_{j} \phi\left(e_{j}\right)\right)$, $j$ is maximal,

where either $j=n$ and the last inequality is an equality, or $j<n$ and the last inequality is strict. In the first case, since $\phi$ is order conserving, it follows that $\phi$ has an ordered decomposition $\phi_{1}^{\prime}+\phi_{2}^{\prime}$, where $\phi_{1}^{\prime}$ is of refinement type; thus, by order irreducibility, $\phi_{1}=\phi_{1}^{\prime}$. We show that the second case cannot hold. This, and induction, completes the proof of the proposition.

Let us illustrate this situation with the following diagram, where $j=4, a_{1}, a_{2}, a_{3}$ are the rank one diagonal projections of $T_{m}$, in order, comprising $Q_{1} \phi\left(e_{1}\right) ; b_{1}, b_{2}, b_{3}$, those of $Q_{2} \phi\left(e_{2}\right) ; c_{1}, c_{2}, c_{3}$, those of 
$Q_{3} \phi\left(e_{3}\right) ;$ and $d_{1}, d_{2}$, those of $Q_{4} \phi\left(e_{4}\right)$ :

$$
\underbrace{a_{1}, a_{2}, a_{3}}_{Q_{1}}, \underbrace{b_{1}, b_{2}, b_{3}}_{Q_{2}}, \underbrace{c_{1}, c_{2}, c_{3}}_{Q_{3}}, \underbrace{d_{1}, d_{2}}_{Q_{4}}, x, \ldots, y, \ldots
$$

Now let $x$ denote the first rank one projection of $T_{m}$ which follows $Q_{j}$ and satisfies $x \phi(1) \neq 0$. Since $\phi$ maps $T_{n}$ into $T_{m}$, the assumption on $j$ implies that $x$ is necessarily a subprojection of $\phi\left(e_{1}\right)$ (and in our illustration could be written as $a_{4}$ ). Also, let $y$ be the first rank one projection of $T_{m}$ such that $y \phi\left(e_{j}\right) \neq 0$ and $y Q_{j}=0$. Such a projection exists since $\phi$ is star extendible and so the projections $\phi\left(e_{1}\right), \ldots, \phi\left(e_{j}\right)$ have equal rank. (In our illustration, $y$ could be written as $d_{3}$.)

We can now see that order conservation is contradicted. For note that $\phi\left(e_{j-1, j}\right)$ maps $y$ into $Q_{j-1}$. Thus, while the partial isometry $v=e_{1}+e_{j-1, j}$ is order conserving, we have $x \prec_{A_{2}} y$ and $\phi(v) y \phi(v)^{*} \prec_{A_{2}}$ $\phi(v) x \phi(v)^{*}$.

Refinement type embeddings $\phi: A_{1} \rightarrow A_{2}$ are determined, uniquely, by their triangular restrictions, $\phi_{t}: A_{1}^{t} \rightarrow A_{2}^{t}$, where $A_{1}^{t} \subseteq A_{1}$ is a maximal triangular subalgebra (isomorphic to $T_{n}$ for some $n$ ) and where $A_{2}^{t} \subseteq A_{2}$ is a similar appropriately chosen triangular subalgebra. Compositions of refinement type embeddings between triangular nest algebras are of refinement type; it follows from this and the remark after Definition 3.3 that general refinement type embeddings are closed under compositions.

Furthermore, refinement type embeddings between triangular algebras are determined up to inner conjugacy by their restrictions to the diagonal subalgebra. Accordingly, it follows (even without the use of Theorem 3.2) that refinement type embeddings $\phi, \psi: A_{1} \rightarrow A_{2}$ are inner conjugate if, and only if, $K_{0} \phi=K_{0} \psi$.

Definition 3.8. A star extendible embedding $\phi: A_{1} \rightarrow A_{2}$ between finite dimensional nest algebras is said to be of $T_{2}$-degenerate type if there exist atomic interval projections $Q, Q^{\prime}$ for $A_{2}$ such that $\phi(1) \leq$ $Q+Q^{\prime}$ 
For an example of an embedding of $T_{2}$-degenerate type, consider the multiplicity two embedding $\phi: T_{4} \otimes M_{m} \rightarrow T_{2} \otimes M_{n}$ for which

$$
\left[\begin{array}{cccc}
a & x & \cdot & \cdot \\
& b & y & \cdot \\
& & c & z \\
& & & d
\end{array}\right] \longrightarrow\left[\begin{array}{llll|llll}
a & & & & x & * & * & \\
& a & x & \cdot & & & & \cdot \\
& & b & y & & & & \cdot \\
& & & c & & & & z \\
\hline & & & & b & y & * & \\
& & & & & c & z & \\
& & & & & & d & \\
& & & & & & & d
\end{array}\right]
$$

Note that $\phi=\theta_{1}+\theta_{2}$ where $\theta_{1}, \theta_{2}$ are rank one embeddings determined, respectively, by the block groupings $(a, b c d)$ and $(a b c, d)$. The presence of $b$ in block two and $c$ in block one show that $\phi$ is not of refinement type. Also $\phi$ is order-irreducible. Since it can be checked that all order irreducible embeddings from $T_{3} \otimes M_{n}$ to $T_{2} \otimes M_{n}$ are of refinement type, $\phi$ can be viewed as the simplest order irreducible non refinement type order conserving embedding.

It can be shown, as in [10], that a $T_{2}$-degenerate embedding is automatically regular. Furthermore, every $T_{2}$-degenerate embedding is, essentially by default, order conserving, and so Theorem 3.2 implies that $T_{2}$-degenerate embeddings have the $K_{0}$-uniqueness property. Since it is illuminating, we give a direct proof of this.

Let $q_{1}, \ldots, q_{p}$ (resp. $Q_{1}, \ldots, Q_{n}$ ) be the atomic interval projections of $A_{1}$ (resp. $A_{2}$ ). Then, for a $T_{2}$-degenerate embedding $\phi$, one sees that $K_{0} \phi$ is an $n \times p$ matrix with at most two nonzero rows, yielding a submatrix

$$
\left[\begin{array}{llll}
u_{1} & u_{2} & \ldots & u_{p} \\
v_{1} & v_{2} & \ldots & v_{p}
\end{array}\right]
$$

for which $v_{i} \leq v_{j}$ and $u_{i} \geq u_{j}$, for $i \leq j$, and $u_{i}+v_{i}=r$, for all $i$, where $r$ is the multiplicity of $\phi$.

The map $\phi: A_{1} \rightarrow A_{2}$ admits a direct sum decomposition into rank one embeddings belonging to $p+1$ distinct inner equivalence classes. The corresponding $K_{0}$ matrices for embeddings in these classes have 
the form

$$
\begin{aligned}
& {\left[\begin{array}{llllll}
1 & 1 & \ldots & 1 & 1 & 1 \\
0 & 0 & \ldots & 0 & 0 & 0
\end{array}\right],} \\
& {\left[\begin{array}{llllll}
1 & 1 & \ldots & 1 & 1 & 0 \\
0 & 0 & \ldots & 0 & 0 & 1
\end{array}\right],} \\
& {\left[\begin{array}{llllll}
1 & 1 & \ldots & 1 & 0 & 0 \\
0 & 0 & \ldots & 0 & 1 & 1
\end{array}\right],} \\
& {\left[\begin{array}{llllll}
0 & 0 & \ldots & 0 & 0 & 0 \\
1 & 1 & \ldots & 1 & 1 & 1
\end{array}\right] .}
\end{aligned}
$$

If there are $r_{k}$ repetitions of the $\mathrm{k}^{\text {th }}$ class then we say that $\phi$ has multiplicity signature $\left\{r_{1}, \ldots, r_{n+1}\right\}$. In this case

$$
K_{0} \phi=\left[\begin{array}{cccc}
r_{1}+r_{2}+\ldots r_{p} & r_{1}+r_{2}+\ldots r_{p-1} & \ldots & r_{1} \\
r_{p+1} & r_{p}+r_{p+1} & \ldots & r_{2}+r_{3}+\ldots r_{p+1}
\end{array}\right]
$$

and so it follows that $K_{0} \phi$ determines the multiplicity signature and thus the inner equivalence class of $\phi$.

When the following theorem is combined with Proposition 3.6, it yields a structure theorem analogous to Proposition 3.7 for order conserving embeddings defined on a nest algebra with no rank one atoms.

Theorem 3.9. Let $\phi: A_{1} \rightarrow A_{2}$ be a regular star extendible embedding between finite dimensional nest algebras which is order conserving and order irreducible, and suppose that $A_{1}$ has no one dimensional atoms. Then $\phi$ is either $T_{2}$-degenerate or is of refinement type.

Proof. Assume that $\phi$ is not $T_{2}$-degenerate. Let $A_{1}$ have atomic interval projections $q_{1}, q_{2}, \ldots, q_{n}$ and let $A_{2}$ have atomic interval projections $Q_{1}, Q_{2}, \ldots, Q_{m}$. The hypothesis for $A_{1}$ implies that there exists a matrix unit system $v_{i j}^{p}, 1 \leq i \leq j \leq n, p=0,1$, for a copy of $T_{n} \otimes \mathbb{C}^{2}$ in $A_{1}$ such that $v_{i j}^{p}$ is supported in $q_{i} A_{1} q_{j}$, the $(i, j)$ block subspace of $A_{1}$. In particular note that for $i<n$ there are rank two order conserving partial isometries in $A_{1}$ of the form $v=v_{i, j_{1}}^{0}+v_{i, j_{2}}^{1}$. Similarly, there are rank two order conserving partial isometries supported in a single block column.

Let $\phi=\theta_{1}+\cdots+\theta_{r}$ be the multiplicity one decomposition of $\phi$, with $r>1$. Note first that since $\phi$ is order conserving, any subsum, such as $\theta_{s}+\theta_{t}$, is also order conserving. Secondly, observe that for each $s$, the partial isometry $\theta_{s}\left(v_{1 n}^{0}\right)$ is supported in a single off-diagonal block. For if not, then $Q_{i} \theta_{s}\left(v_{1 n}^{0}\right) Q_{i}=\theta_{s}\left(v_{1 n}^{0}\right)$ for some $s$ and $i$, and so the range of $\theta_{s}$ lies in $Q_{i} A_{2} Q_{i}$. On the other hand $\phi$ is order conserving and 
it follows readily that $\theta_{s}$ must be an order summand of $\phi$, and hence equal to $\phi$ by order irreducibility, contrary to the assumption $r>1$.

Consider an index $t$ for which $\theta_{t}\left(v_{1 n}^{0}\right)$ is supported in the $(k, l)$ block subspace and is such that $Q_{i} \phi\left(v_{1 n}^{0}\right) Q_{j}=0$ for all $(i, j) \neq(k, l)$ satisfying $k \leq i$ and $j \leq l$. We complete the proof of the lemma by showing that for all $s, i, j$ the partial isometry $\theta_{s}\left(v_{i j}^{0}\right)$ is supported in the same block subspace as $\theta_{t}\left(v_{i j}^{0}\right)$. In particular all the summands $\theta_{s}$ are inner conjugate and $\phi$ is a refinement embedding.

Note first that $\theta_{t}\left(v_{11}^{0}\right)$ is supported in the $Q_{k}$ block subspace and that $\theta_{t}\left(v_{n n}^{0}\right)$ is supported in the $Q_{l}$ block subspace. Given $s \neq t$, there are various a priori possibilities for the support projections $Q_{i}$ of $\theta_{s}\left(v_{11}^{0}\right)$. The first possibility, $i<j$, is suggested by the following diagram:

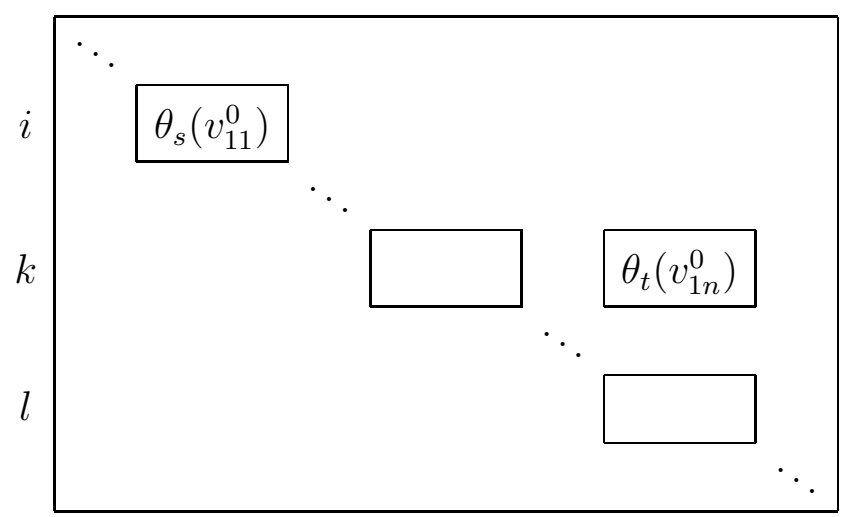

In this case we can deduce from the order conserving nature of $\phi$ that $\theta_{s}\left(v_{n n}^{0}\right)$ is supported in a block $Q_{j}$ with $j \leq k$. Indeed, if such a $j$ is greater than $k$, then it follows that $\theta_{s}\left(v_{1 n}^{0}\right)$ and $\theta_{s}\left(v_{1 n}^{1}\right)$ have support in the $(i, j)$ block, $\theta_{t}\left(v_{11}^{0}\right)$ and $\theta_{t}\left(v_{11}^{1}\right)$ have support in the $(k, k)$ block and so the support of $\theta_{s}\left(v_{1 n}^{0}\right)+\theta_{t}\left(v_{11}^{1}\right)$ is not of staircase type. Since $\theta_{s}+\theta_{t}$ is order conserving, this is a contradiction.

We have shown that if $i<k$ then $\theta_{s}+\theta_{t}$ is an ordered sum. Now suppose that $i \geq l$. Since $Q_{i}$ is the support projection for $\theta_{s}\left(v_{11}^{0}\right)$, it follows immediately (without the need for the order conservation of $\phi$ ) that $\theta_{t}+\theta_{s}$ is an ordered sum.

Now consider the case $k<i<l$. Then $\theta_{s}\left(v_{11}^{1}\right)+\theta_{t}\left(v_{1 n}^{0}\right)$ has support in the $(i, i)$ block and the $(k, l)$ block and so is not of staircase form. Since $\phi$ is order conserving, we have a contradiction once again. Thus the only possible value for $i$ is $k$.

In summary, we have shown that if $s \neq t$, then $\theta_{s}\left(v_{11}^{0}\right)$ is supported in the same block $Q_{k}$ as $\theta_{t}\left(v_{11}^{0}\right)$. By the original assumption on $t$, we must have that $\theta_{s}\left(v_{1 n}^{0}\right)$ is supported in the $(k, l)$ block, since the only alternative is that it is supported in a $(k, r)$ with $r>l$. But this would imply that $\left(\theta_{s}+\theta_{t}\right)\left(v_{n n}^{0}+v_{1 n}^{1}\right)$ is not order conserving. 
For similar reasons it follows that for $1<x<n$, the projections $\theta_{s}\left(v_{x x}^{0}\right)$ and $\theta_{t}\left(v_{x x}^{0}\right)$ are equivalent. For suppose that these projections are supported in the blocks for $Q_{p}$ and $Q_{q}$ respectively, with $p<q$. Then $\theta_{s}\left(v_{x n}^{0}\right)$ has support in the $(p, l)$ block while $\theta_{t}\left(v_{x x}^{1}\right)$ has support in the $(q, q)$ block and hence $\theta_{s}+\theta_{t}$ is not order conserving. Similarly, $q<p$ is not possible.

We have shown that for all $1 \leq x \leq n$ and for all $s$ and $t$ the projections $\theta_{s}\left(v_{x x}^{0}\right)$ and $\theta_{t}\left(v_{x x}^{0}\right)$ lie in the same block subspace and so are inner equivalent. It follows that $\phi$ is a refinement type embedding.

Corollary 3.10. Let $\phi: A_{1} \rightarrow A_{2}$ be an order conserving embedding between finite dimensional nest algebras, where $A_{1}$ has no rank one atoms. Then $\phi$ can be written as an ordered sum $\phi=\phi_{1}+\cdots+\phi_{p}$, where each $\phi_{i}$ is either $T_{2}$-degenerate or of refinement type.

Remark. Theorem 3.9 is not valid if $A_{1}$ has both rank one atoms and higher rank atoms. There are examples of embeddings which are order conserving and order irreducible but neither $T_{2}$-degenerate nor of refinement type. For example, let $\phi: T(2,2,1) \longrightarrow T(6,3,1)$ be given by

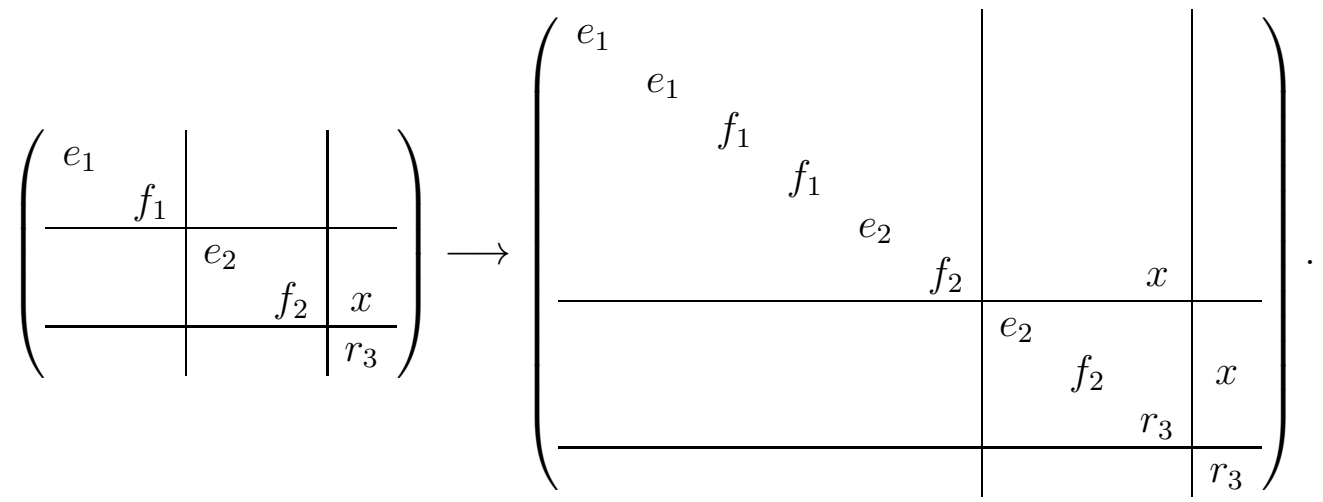

Only one off diagonal position has been indicated; all off diagonal matrix units are dealt with in the same way. Their image under $\phi$ matches the diagonal matrix units of the domain and the range spaces in the order in which they appear on the diagonal.

Note that in this example, if $\phi$ is restricted to the orthogonal complement of the rank one atom of $A_{1}$, then it is no longer order irreducible.

It is easy to construct a variety of similar counterexamples in which the number of atoms in the codomain which intersect $\phi(1)$ is arbitrarily large.

3.2. Order preserving embeddings. Donsig [2] has extended the notion of order preservation to the general context of digraph algebras 
(where order preservation is defined with respect to a self-adjoint subalgebra of the digraph algebra). His definition, when restricted to a finite dimensional nest algebra and taken with respect to $A \cap A^{*}$, agrees with the definition below.

Definition 3.11. Let $A \subseteq M_{n}$ be a finite dimensional nest algebra with atomic interval projections $q_{1}, q_{2}, \ldots, q_{l}$.

1. The set $\operatorname{Pisom}_{\mathrm{op}}(A)$ is the set of partial isometries $v$ in $\operatorname{Pisom}_{\mathrm{reg}}(A)$ which are order preserving in the sense that

$q_{i} v q_{j} \neq 0 \Longrightarrow q_{s} v q_{t}=0, \quad$ for $(s, t) \neq(i, j)$ with $s \leq i$ and $t \geq j$.

2. A star extendible embedding between finite dimensional nest algebras is order preserving if it maps order preserving partial isometries to order preserving partial isometries.

3. A star extendible embedding $\phi: A_{1} \rightarrow A_{2}$ is locally order preserving if $\phi(v)$ is in $\operatorname{Pisom}_{\mathrm{op}}\left(A_{2}\right)$, for each rank one element $v$ in $\operatorname{Pisom}_{\text {reg }}\left(A_{1}\right)$.

For triangular nest algebras $T_{n}, n=1,2, \ldots$, the order preserving embeddings coincide with the order conserving embeddings, since a partial isometry in $T_{n}$ is order conserving if, and only if, it is order preserving. In contrast, for non-triangular algebras order preservation is quite stringent. For example, a star extendible embedding,

$$
\phi: T_{2} \rightarrow T_{2} \otimes M_{n},
$$

is order preserving if, and only if, either

$$
\phi\left(e_{12}\right) \in D_{2} \otimes M_{n} \quad \text { or } \quad \phi\left(e_{12}\right) \in e_{12} \otimes M_{n} .
$$

On the other hand, if $\phi: T_{n} \rightarrow T_{m}$ is an order conserving embedding and $\eta: B_{1} \rightarrow B_{2}$ is a $\mathrm{C}^{*}$-algebra homomorphism between finite dimensional $\mathrm{C}^{*}$-algebras, then the star extendible homomorphism,

$$
\phi \otimes \eta: T_{n} \otimes B_{1} \rightarrow T_{m} \otimes B_{2},
$$

has partial embeddings (between the summands of the algebras) which are locally order preserving. Although the local order preservation property is not generally conserved under composition, this is plainly the case for these tensor product maps.

We remark that there are order conserving embeddings which are locally order preserving but are not order preserving. The multiplicity two standard embedding from $T_{3} \otimes M_{r}$ to $T(r, r, 2 r, r, r)$ has this property.

It is also possible for an embedding to be order preserving but not order conserving. To see this consider the map $T_{2} \otimes M_{2} \rightarrow T_{4} \otimes M_{2}$ 
given by

$$
\left(\begin{array}{l|l}
a & b \\
\hline & c
\end{array}\right) \rightarrow\left(\begin{array}{l|l|l|l}
a & & b & \\
\hline & a & & b \\
\hline & & c & \\
\hline & & & c
\end{array}\right) .
$$

Finally, the embedding $T_{2} \rightarrow T_{2} \otimes M_{3}$ given by

$$
\left(\begin{array}{ll}
a & b \\
& c
\end{array}\right) \longrightarrow\left(\begin{array}{ccc|cc}
a & b & & & \\
& c & & & \\
& & a & b & \\
\hline & & c & & \\
& & & a & b \\
& & & & c
\end{array}\right)
$$

illustrates how easily a 'nice' embedding can fail to be locally order preserving. (For embeddings with domain $T_{2}$, order preservation and local order preservation coincide.)

\section{ISOMORPHISMS}

Let $\mathcal{A}=\left\{A_{k}, \alpha_{k}\right\}, \mathcal{A}^{\prime}=\left\{A_{k}^{\prime}, \alpha_{k}^{\prime}\right\}$ be direct systems of digraph algebras with embeddings $\alpha_{k}, \alpha_{k}^{\prime}$ which are star extendible and regular. Let $A_{0}, A_{0}^{\prime}$ be the locally finite algebras which are the algebraic direct limits of the systems. The limit algebras $A_{0}, A_{0}^{\prime}$ are said to be star extendibly isomorphic if there is an algebra isomorphism $\Phi_{0}: A_{0} \rightarrow A_{0}^{\prime}$ which extends to $\mathrm{a}^{*}$-algebra isomorphism between the generated $\mathrm{C}^{*}$ algebras.

Definition 4.1. The direct systems $\mathcal{A}, \mathcal{A}^{\prime}$ are said to be regularly isomorphic if there exist regular, star extendible embeddings $\left\{\phi_{k}, \psi_{k}\right\}$ such that the diagram

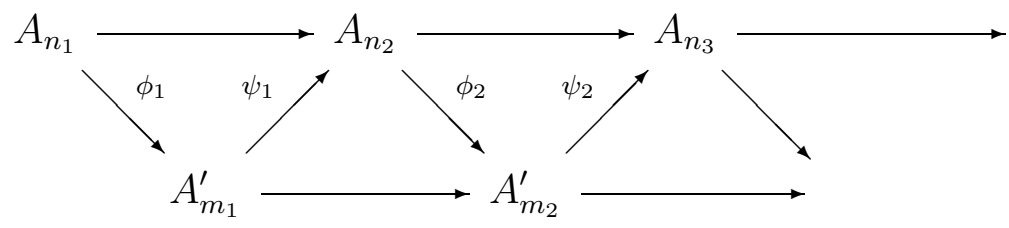

commutes.

A fundamental issue in the classification of nonselfadjoint limit algebras is that a star extendible isomorphism $\Phi: A_{0} \rightarrow A_{0}^{\prime}$ need not be induced by a regular isomorphism between the given systems. (See [4, 21].) However, we shall see that for various order conserving systems this is indeed the case. This enables us to define invariants for limit algebras in terms of regular isomorphism invariants for systems. 
In the proof of the next lemma the block matrix support of an element $a$ in a finite dimensional nest algebra refers to the set of block subspaces $Q_{i} A Q_{j}$ for which $Q_{i} a Q_{j} \neq 0$. The matrix support of $a$ refers to a given matrix unit system $\left\{h_{i j}\right\}$ and is the set $\left\{(i, j) \mid h_{i i} a h_{j j} \neq 0\right\}$.

Lemma 4.2. Let $\phi: A_{1} \rightarrow A_{2}, \psi: A_{2} \rightarrow A_{3}$ be star extendible homomorphisms between finite dimensional nest algebras such that the composition $\psi \circ \phi$ is locally order conserving. If $h$ is a rank one regular partial isometry in $\operatorname{Pisom}_{\text {reg }}\left(A_{2}\right)$ which lies in the bimodule over $A_{2} \cap A_{2}^{*}$ generated by $\phi\left(A_{1}\right)$, then $\psi(h)$ is an order conserving partial isometry.

Proof. Fix matrix units for $A_{1}$ and $A_{2}$ so that $\phi$ maps matrix units of $A_{1} \cap A_{1}^{*}$ to sums of matrix units in $A_{2} \cap A_{2}^{*}$, and then choose matrix units for $A_{3}$ such that $\psi$ maps matrix units in $A_{2} \cap A_{2}^{*}$ to sums of matrix units. Let $B$ be the bimodule over $A_{2} \cap A_{2}^{*}$ generated by $\phi\left(A_{1}\right)$. Since $B$ is, in particular, a bimodule over the diagonal matrices, $B$ is the linear span of the matrix units which it contains. Let $e$ be a matrix unit in $B$. Then there are atoms $P$ and $Q$ for $A_{2}$ such that $e=P e Q$. If $f$ and $g$ are matrix units satisfying $f=\operatorname{PfP}$ and $g=Q g Q$, then $f, g \in A_{2} \cap A_{2}^{*}$. It follows that $f e g \in B$. But any matrix unit $e^{\prime}$ for which $e^{\prime}=P e^{\prime} Q$ can be written in this form. Thus, if $P B Q \neq\{0\}$ then $P B Q=P A_{2} Q$. This enables us to identify the bimodule over $A_{2} \cap A_{2}^{*}$ generated by $\phi\left(A_{1}\right)$ : it consists of all elements of $A_{2}$ which are supported in the collection of matrix blocks which contain non-zero entries for elements of $\phi\left(A_{1}\right)$.

Now suppose that $h$ and $e$ are two matrix units in the same block in $A_{2}$ and suppose, further, that $\psi(e)$ is order conserving. By the first assumption, there are matrix units $f$ and $g$ in diagonal blocks of $A_{2}$ such that $h=f e g$. Since $\psi(e)$ is order conserving, its support has 'staircase' form in $A_{3}$; since $\psi(f)$ and $\psi(g)$ lie in $A_{3} \cap A_{3}^{*}, \psi(h)$ also has 'staircase' form; i.e. $\psi(h)$ is also order conserving. This argument works equally well if $h$ is a rank one partial isometry in the same block as $e$ rather than a matrix unit. (The only modification is that $f$ and $g$ are merely rank one partial isometries in the appropriate diagonal blocks.) As a consequence of these observations, we see that we can prove the lemma by proving it in the special case in which $h$ is a subordinate of a partial isometry in the range, $\phi\left(A_{1}\right)$, of $\phi$,

If we make this additional hypothesis, there is a rank one regular partial isometry $e$ in $A_{1}$ such that $h h^{*} \phi(e) h^{*} h \neq 0$. Because of our matrix unit choice for $\psi$, it follows that the matrix support of $\psi(\phi(e))$ contains the matrix support of $\psi(h)$. However, the block matrix support of $\psi(\phi(e))$ is of staircase type, since $\psi \circ \phi$ is locally order conserving. It follows that $\psi(h)$ is an order conserving partial isometry. 
Remark. The assumption that $\psi \circ \phi$ is locally order conserving does not imply that $\psi$ is locally order conserving, even when $\psi$ is restricted to the algebra generated by $\phi\left(A_{1}\right)$ and $A_{2} \cap A_{2}^{*}$. The two embeddings $\phi: T_{2} \rightarrow T(1,2,1)$ and $\psi: T(1,2,1) \rightarrow T(1,1,4,1,1)$ given by

$$
\left(\begin{array}{l|l}
a & b \\
\hline & c
\end{array}\right) \stackrel{\phi}{\longrightarrow}\left(\begin{array}{l|l|l|l}
a & b & & \\
\hline & c & & \\
& & a & b \\
\hline & & & c
\end{array}\right)
$$

and

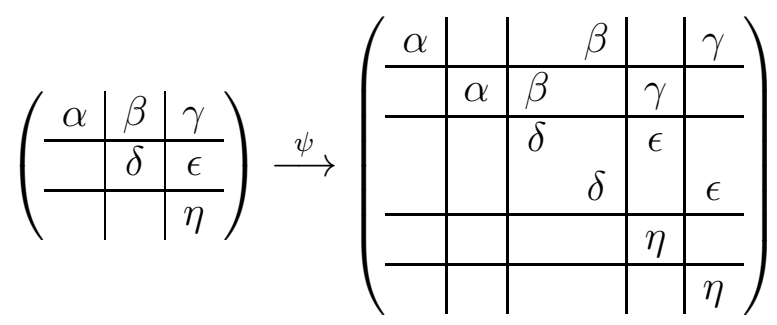

provide a counter-example. Note that $T(1,2,1)$ is the algebra generated by $\phi\left(T_{2}\right)$ and $T(1,2,1) \cap T(1,2,1)^{*}$.

Theorem 4.3. Let $\mathcal{A}, \mathcal{A}^{\prime}$ be systems of finite dimensional nest algebras in which all compositions of embeddings are locally order conserving and let $A_{0}, A_{0}^{\prime}$ be their algebraic limit algebras. Then the following are equivalent.

(i) $\mathcal{A}$ and $\mathcal{A}^{\prime}$ are regularly isomorphic.

(ii) $A_{0}$ and $A_{0}^{\prime}$ are star extendibly isomorphic.

Moreover, every star extendible isomorphism $\Phi: A_{0} \rightarrow A_{0}^{\prime}$ is induced by a regular star extendible isomorphism between the systems $\mathcal{A}, \mathcal{A}^{\prime}$.

Proof. The proof that (i) implies (ii) is immediate. For the converse, let $\Phi: A_{0} \rightarrow A_{0}^{\prime}$ be a star extendible isomorphism. Since each algebra in the systems $\mathcal{A}$ and $\mathcal{A}^{\prime}$ is finitely generated, there is a commuting diagram isomorphism of the systems with crossover maps $\phi_{k}, \psi_{k}$ :

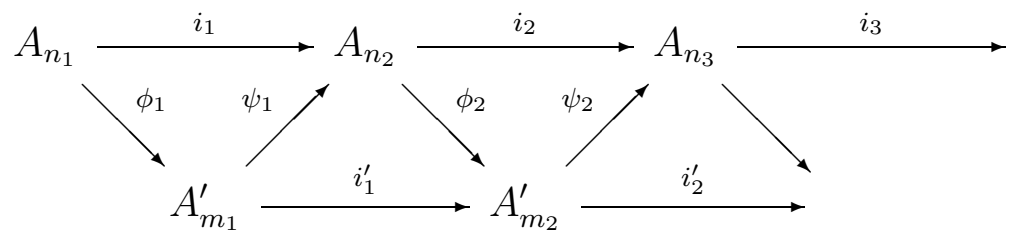

Since $i_{1}=\psi_{1} \circ \phi_{1}$, the range of $i_{1}$ is contained in $\psi\left(A_{m_{1}}^{\prime}\right)$. By hypothesis, $i_{1}$ is locally order conserving and so locally regular and hence regular. If $x \in A_{n_{1}}$ is a rank one regular partial isometry, then $i_{1}(x)$ is a regular, order conserving, partial isometry in $\psi\left(A_{m_{1}}^{\prime}\right)$. Consequently, 
$i_{1}(x)$ is a sum, $i_{1}(x)=y_{1}+\cdots+y_{s}$, of regular rank one partial isometries in $\psi\left(A_{m_{1}}^{\prime}\right)$. Since $i_{1}^{\prime}=\phi_{2} \circ \psi_{1}$ is locally order conserving, each partial isometry $\phi_{2}\left(y_{j}\right)$ is order conserving, by Lemma 4.2, and hence regular in $A_{m_{2}}^{\prime}$. Therefore $\phi_{2} \circ i_{1}(x)$ is regular and so $\phi_{2} \circ i_{1}: A_{n_{1}} \rightarrow A_{m_{2}}^{\prime}$ is locally regular. By Theorem 2.4, $\phi_{2} \circ i_{1}=\phi_{2} \circ \psi_{1} \circ \phi_{1}$ is a regular embedding.

The same argument shows that $\psi_{3} \circ \phi_{3} \circ \psi_{2}=\psi_{3} \circ i_{2}^{\prime}$ is regular; continuing in this fashion, we may replace the initial commuting diagram isomorphism with one whose crossover maps are all regular. This shows that (ii) implies (i).

The last theorem is important in that it shows that tentative invariants defined for algebraic limit algebras in terms of specific regular presentations do not in fact depend on the presentation and are thus genuine invariants for star extendible isomorphism. In the next section we shall need to know that certain scales defined in terms of order preserving or order conserving systems are invariants. This will follow from the following refinement of Theorem 4.3 in the order preserving or order conserving cases.

Theorem 4.4. Let $\mathcal{A}, \mathcal{A}^{\prime}$ be systems of finite dimensional nest algebras in which all embeddings are order preserving (resp. order conserving) and let $A_{0}, A_{0}^{\prime}$ be their algebraic limit algebras. Then the following are equivalent.

(i) The systems $\mathcal{A}$ and $\mathcal{A}^{\prime}$ are regularly isomorphic by a commuting diagram isomorphism in which all the crossover maps are order preserving (resp. order conserving).

(ii) $A_{0}$ and $A_{0}^{\prime}$ are star extendibly isomorphic.

Moreover, every star extendible isomorphism $\Phi: A_{0} \rightarrow A_{0}^{\prime}$ is induced by a system isomorphism as in (河).

Proof. The proof of this theorem follows easily from Lemma 4.5 below. If $A_{0}$ and $A_{0}^{\prime}$ are star extendibly isomorphic, then Theorem 4.3 gives a regular system isomorphism in which any adjacent pair of crossover maps have a composition which is order preserving or order conserving, as appropriate. From the lemma, any consecutive triple of crossover maps has composition which is order preserving or order conserving; a sequence of such triple compositions yields a system isomorphism with order preserving or order conserving crossover maps.

Lemma 4.5. Let

$$
A_{1} \stackrel{\phi}{\longrightarrow} A_{2} \stackrel{\psi}{\longrightarrow} A_{3} \stackrel{\eta}{\longrightarrow} A_{4}
$$


be regular star extendible homomorphsims between finite dimensional nest algebras such that the compositions $\psi \phi$ and $\eta \psi$ are order preserving (resp. order conserving). Then the triple composition $\eta \psi \phi$ is order preserving (resp. order conserving).

Before giving a proof of the Lemma, we discuss some helpful preliminary matters. When $A_{1}$ is a finite dimensional nest, we number the block rows and block columns of $A_{1}$ in the natural way. If $v \in A_{1}$ is supported in a single block, $\operatorname{row}(v)$ and $\operatorname{col}(v)$ denote the row and column in which the support block is located. Suppose that $\phi: A_{1} \rightarrow A_{2}$ is a multiplicity one (star extendible, regular) embedding of $A_{1}$ into another finite dimensional nest algebra. If $v$ is supported in a single block in $A_{1}$, then $\phi(v)$ is supported in a single block in $A_{2}$. If $v$ and $w$ are each supported in a single block, then

$$
\begin{aligned}
\operatorname{row}(v)=\operatorname{row}(w) & \Longrightarrow \operatorname{row}(\phi(v))=\operatorname{row}(\phi(w)), \\
\operatorname{col}(v)=\operatorname{col}(w) & \Longrightarrow \operatorname{col}(\phi(v))=\operatorname{col}(\phi(w)), \\
\operatorname{row}(v)<\operatorname{row}(w) & \Longrightarrow \operatorname{row}(\phi(v)) \leq \operatorname{row}(\phi(w)), \\
\operatorname{col}(v)<\operatorname{col}(w) & \Longrightarrow \operatorname{col}(\phi(v)) \leq \operatorname{col}(\phi(w)) .
\end{aligned}
$$

In particular, if $v$ and $w$ are supported in the same block, then so are $\phi(v)$ and $\phi(w)$. Thus, $\phi$ induces a map $\tilde{\phi}$ from the blocks of $A_{1}$ to the blocks of $A_{2}$. This map need not be injective, but it does respect the block structure: if $\operatorname{row}(X)<\operatorname{row}(Y)$, then $\operatorname{row}(\tilde{\phi}(X)) \leq \operatorname{row}(\tilde{\phi}(Y))$; similarly, if $\operatorname{col}(X)<\operatorname{col}(Y)$, then $\operatorname{col}(\tilde{\phi}(X)) \leq \operatorname{col}(\tilde{\phi}(Y))$. (Here, $X$ and $Y$ denote blocks in $A_{1}$, not elements of the nest algebra.) In particular, if $X$ and $Y$ are blocks in the same row (or column), then $\tilde{\phi}(X)$ and $\tilde{\phi}(Y)$ are also blocks in the same row (or column).

Let $v$ and $w$ be partial isometries each of which is supported in a single block and assume that $v+w$ is a partial isometry which is not order preserving. Then the block support for one of the partial isometries, say $v$, is located to the "southwest" of the block support for the other partial isometry. More precisely,

$$
\operatorname{row}(w) \leq \operatorname{row}(v) \text { and } \operatorname{col}(v) \leq \operatorname{col} w,
$$

with at least one inequality being strict. This can be indicated by the diagrams:

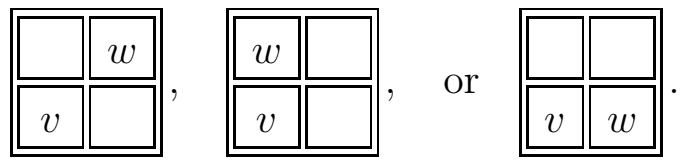

Now suppose that $\phi(v+w)=\phi(v)+\phi(w)$ is order preserving. (The assumption that $\phi$ has multiplicity one is still in force.) It follows from 
the relations above that the block support for $\phi(v)$ is to the "southwest," i.e., that

$$
\operatorname{row} \phi(w) \leq \operatorname{row} \phi(v) \text { and } \operatorname{col} \phi(v) \leq \operatorname{col} \phi(w) .
$$

But $\phi(v)+\phi(w)$ is order preserving, so in fact row $\phi(v)=$ row $\phi(w)$ and $\operatorname{col} \phi(v)=\operatorname{col} \phi(w)$; i.e., $\phi(v)$ and $\phi(w)$ have the same block support.

Now assume that $\phi: A_{1} \rightarrow A_{2}$ is an embedding with arbitrary multiplicity. Suppose that $\phi$ is not order preserving. Then one (or both) of two situations must occur. The first is that there is a rank one partial isometry $v \in A_{1}$ and a pair of multiplicity one summands $\phi_{1}$ and $\phi_{2}$ of $\phi$ such that $\phi_{1}(v)+\phi_{2}(v)$ is not order preserving. The other alternative is that there are two rank one partial isometries $v$ and $w$ in $A_{1}$ with support in distinct blocks such that $v+w$ is order preserving and there are two multiplicity one summands $\phi_{1}$ and $\phi_{2}$ of $\phi$ such that $\phi_{1}(v)+\phi_{2}(w)$ is not order preserving.

If, on the other hand, $\phi$ is order preserving, then any (partial) sum of the multiplicity one summands for $\phi$ is also order preserving. Likewise, if $\phi$ is order conserving, then any (partial) sum of the multiplicity one summands for $\phi$ is also order conserving.

Note also that a multiplicity one summand of a composition of two embeddings is the composition of multiplicity one summands of each factor.

Proof of Lemma 4.5. The proof of the Lemma in the order preserving context differs considerably from the proof in the order conserving context, so we present the two arguments separately. We start with the the order preserving context, where the argument is simpler.

First, suppose that $v$ is a rank one partial isometry in $A_{1}$ such that $\eta \psi \phi(v)$ is not order preserving. Then there are multiplicty one summands $\phi_{1}$ and $\phi_{2}$ of $\phi, \psi_{1}$ and $\psi_{2}$ of $\psi$, and $\eta_{1}$ and $\eta_{2}$ of $\eta$ such that $\eta_{1} \psi_{1} \phi_{1}(v)+\eta_{2} \psi_{2} \phi_{2}(v)$ is not order preserving.

Observe that $\phi_{1}(v)+\phi_{2}(v)$ is a partial isometry which is not order preserving. It is a partial isometry since $\phi_{1}+\phi_{2}$ is an embedding; if it were order preserving, then its image under $\eta_{1} \psi_{1}+\eta_{2} \psi_{2}$ would be order preserving. But $\eta_{1} \psi_{1} \phi_{1}(v)+\eta_{2} \psi_{2} \phi_{2}(v)$ is a subordinate of this image and is not order preserving.

Since $\psi_{2} \phi_{1}+\psi_{2} \phi_{2}$ (a sum of multiplicity one summands of $\psi \phi$ ) is order preserving, it follows that $\psi_{2} \phi_{1}(v)+\psi_{2} \phi_{2}(v)$ is order preserving. Consequently, $\psi_{2} \phi_{1}(v)$ and $\psi_{2} \phi_{2}(v)$ are in the same block. This, in turn, implies that $\eta_{2} \psi_{2} \phi_{1}(v)$ and $\eta_{2} \psi_{2} \phi_{2}(v)$ are in the same block.

This means that

$$
\eta_{1} \psi_{1} \phi_{1}(v)+\eta_{2} \psi_{2} \phi_{1}(v)
$$


and

$$
\eta_{1} \psi_{1} \phi_{1}(v)+\eta_{2} \psi_{2} \phi_{2}(v)
$$

have the same block structure - either both are order preserving or both are not order preserving. But the second one is not order preserving by assumption while the first one is the image of the rank one partial isometry $\phi_{1}(v)$ under the order preserving embedding $\eta_{1} \psi_{1}+\eta_{2} \psi_{2}$, a contradiction.

The other possibility which we need to consider is that there are rank one partial isometries $v$ and $w$ in $A_{1}$ such $v+w$ is an order preserving partial isometry but $\eta \psi \phi(v+w)$ is not order preserving. Again, there are multiplicty one summands $\phi_{1}$ and $\phi_{2}$ of $\phi, \psi_{1}$ and $\psi_{2}$ of $\psi$, and $\eta_{1}$ and $\eta_{2}$ of $\eta$ such that $\eta_{1} \psi_{1} \phi_{1}(v)+\eta_{2} \psi_{2} \phi_{2}(w)$ is not order preserving.

Observe that $\phi_{1}(v)+\phi_{2}(w)$ is a partial isometry which is not order preserving. It is a partial isometry since it is a subordinate of $\phi(v+w)$; if it were order preserving, then its image under $\eta_{1} \psi_{1}+\eta_{2} \psi_{2}$ would be order preserving. But $\eta_{1} \psi_{1} \phi_{1}(v)+\eta_{2} \psi_{2} \phi_{2}(w)$ is a subordinate of this image and is not order preserving.

Since $\psi_{2} \phi_{1}+\psi_{2} \phi_{2}$ is order preserving, it follows that $\psi_{2} \phi_{1}(v)+$ $\psi_{2} \phi_{2}(w)$ is order preserving. Consequently, $\psi_{2} \phi_{1}(v)$ and $\psi_{2} \phi_{2}(w)$ are in the same block, whence $\eta_{2} \psi_{2} \phi_{1}(v)$ and $\eta_{2} \psi_{2} \phi_{2}(v)$ are in the same block.

This means that

$$
\eta_{1} \psi_{1} \phi_{1}(v)+\eta_{2} \psi_{2} \phi_{1}(w)
$$

and

$$
\eta_{1} \psi_{1} \phi_{1}(v)+\eta_{2} \psi_{2} \phi_{2}(w)
$$

have the same block structure But $\eta_{1} \psi_{1} \phi_{1}(v)+\eta_{2} \psi_{2} \phi_{2}(w)$ is not order preserving by assumption while $\eta_{1} \psi_{1} \phi_{1}(v)+\eta_{2} \psi_{2} \phi_{1}(w)$ is a subordinate of the image of the order preserving partial isometry $\phi_{1}(v+w)$ under the order preserving embedding $\eta_{1} \psi_{1}+\eta_{2} \psi_{2}$, a contradiction. $\left(\phi_{1}(v+\right.$ $w$ ) is order preserving since any multiplicity one embedding is order preserving and $v+w$ is an order preserving partial isometry.)

Having completed the order preserving context, we move on to the order conserving context. Let $v \in A_{1}$ be an order conserving partial isometry such that the block support of $v$ has staircase form. Let $v^{\prime}=v_{a}+v_{b}$ be a rank two subordinate of $v$ which is supported in two of these block subspaces, denoted $a$ and $b$. The orientation of these blocks in the block decomposition of $A_{1}$ can be indicated diagramatically as 
one of six types, namely the triple,
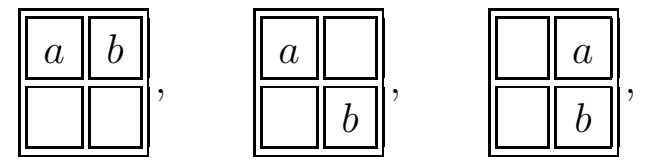

together with the corresponding triple with the letters reversed.

Suppose the $\phi\left(v^{\prime}\right)$ is not order conserving. Then there exist two blocks of $A_{2}, X$ and $Y$ say, which have nonstaircase orientation

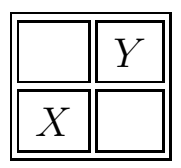

and are such that at least one of four possibilities occurs:

(i) $\phi\left(v_{a}\right)$ has support meeting $X$ and $Y$

(ii) $\phi\left(v_{b}\right)$ has support meeting $X$ and $Y$

(iii) $\phi\left(v_{a}\right)$ and $\phi\left(v_{b}\right)$ have support meeting $X$ and $Y$ respectively

(iv) $\phi\left(v_{a}\right)$ and $\phi\left(v_{b}\right)$ have support meeting $Y$ and $X$ respectively.

Consider the companion blocks $U, V$ for $X, Y$ indicated by the diagram

$$
\begin{array}{|l|l|}
\hline U & Y \\
\hline X & V \\
\hline
\end{array}
$$

Since the composition $\psi \phi$ is order conserving, if $\psi_{1}$ is a multiplicity one summand of $\psi$, the composition $\psi_{1} \phi$ is order conserving. In particular such a map $\psi_{1}$ must 'correct' the $X, Y$ support of $\phi(v)$ (indicated in one of the four possibilities (i) to (iv)) by a 'fusion' of blocks, as indicated in each of the three diagrams

$$
\begin{array}{|c|c|}
\hline U & Y \\
\hline \hline X & V \\
\hline
\end{array} \longrightarrow \begin{array}{|ll|}
\hline \tilde{\psi}_{1}(U) & \tilde{\psi}_{1}(Y) \\
\hline \tilde{\psi}_{1}(X) & \tilde{\psi}_{1}(V) \\
\hline
\end{array}
$$

\begin{tabular}{|c|c|c|c|c|}
\hline$U$ & $Y$ & & $\tilde{\psi}_{1}(U)$ & $\tilde{\psi}_{1}(Y)$ \\
\hline$X$ & $V$ & & $\tilde{\psi}_{1}(X)$ & $\tilde{\psi}_{1}(V)$ \\
\hline$U$ & $Y$ & & $\tilde{\psi}_{1}(U)$ & $\tilde{\psi}_{1}(Y)$ \\
\hline$X$ & $V$ & & $\tilde{\psi}_{1}(X)$ & $\tilde{\psi}_{1}(V)$ \\
\hline
\end{tabular}

This means that, in the first case for example, the image under $\psi_{1}$ of an element in $A_{2}$ with support in the blocks $U, Y$ or in the blocks $X$, $V$ has support in a single block of $A_{3}$. (The appearance of $\tilde{\psi}_{1}(U)$ and $\tilde{\psi}_{1}(Y)$, for example, in the same block in the diagram indicates that 
$\tilde{\psi}_{1}(U)=\tilde{\psi}_{1}(Y)$.) These types of fusion may vary among the various summands $\psi_{1}$ of $\psi$. The comments preceding the statement of the lemma preclude a correction of the $X, Y$ support of $\phi(v)$ of the form

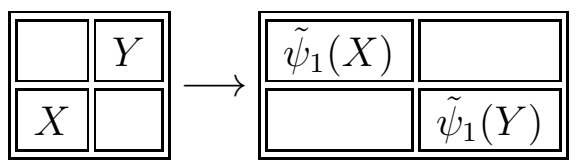

or the similar correction with $\tilde{\psi}_{1}(X)$ and $\tilde{\psi}_{1}(Y)$ interchanged.

Let $w, u \in A_{2}$ be rank two partial isometries with support in the blocks $X, Y$ and $U, V$ respectively. We now show that $\eta \psi(w)$ is order conserving. The idea for this argument is that the fusion of blocks 'binds' the supports of $\eta \psi(w)$ and $\eta \psi(u)$ and the latter is order conserving by the hypotheses, since $u$ is order conserving. More precisely let $w=w_{X}+w_{Y}$ be the rank one decomposition and suppose that $\eta \psi(w)$ is not order conserving. Then there exist two blocks $W, Z$ in $A_{4}$ with nonstaircase form

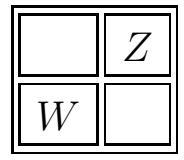

and there exist multiplicity one summands $\psi_{1}, \psi_{2}$ of $\psi$ and $\eta_{1}, \eta_{2}$ of $\eta$ such that at least one of the following possibilities occurs.

(a) $\eta_{1} \psi_{1}\left(w_{X}\right)$ and $\eta_{2} \psi_{2}\left(w_{X}\right)$ meet $W$ and $Z$ respectively.

(b) $\eta_{1} \psi_{1}\left(w_{Y}\right)$ and $\eta_{2} \psi_{2}\left(w_{Y}\right)$ meet $W$ and $Z$ respectively.

(c) $\eta_{1} \psi_{1}\left(w_{X}\right)$ and $\eta_{2} \psi_{2}\left(w_{Y}\right)$ meet $W$ and $Z$ respectively.

(d) $\eta_{1} \psi_{1}\left(w_{X}\right)$ and $\eta_{2} \psi_{2}\left(w_{Y}\right)$ meet $Z$ and $W$ respectively.

Let $u=u_{U}+u_{V}$ be the rank one decomposition of $u$. If the first possibility (a) occurs, then noting that $\psi_{1}$ fuses $X$ and $U$, or $X$ and $V$, and that $\psi_{2}$ also fuses $X$ and $U$, or $X$ and $V$, it follows that $\eta_{1} \psi_{1}\left(u_{U}\right)$ (or $\eta_{1} \psi_{1}\left(u_{V}\right)$ ) meets $W$ and similarly that $\eta_{2} \psi_{2}\left(u_{U}\right)$ (or $\eta_{2} \psi_{2}\left(u_{V}\right)$ ) meets $Z$. For each of these four alternatives $\eta_{1} \psi_{1}(u)+\eta_{2} \psi_{2}(u)$ is not order conserving, and this contradicts the fact that $\eta \psi$ is order conserving. The other three possibilities (b), (c), (d) also lead to contradictions in the same manner.

It has been shown then that $\eta \psi(w)$ is order conserving or, more intuitively, that the map $\eta \psi$ 'corrects' the $X, Y$ block structure of $\phi\left(v^{\prime}\right)$ and $\phi(v)$. We now wish to deduce that since every such block pair is corrected by $\eta \psi$ then in fact $\eta \psi \phi(v)$ is order conserving. This will complete the proof.

To see this, suppose that $\eta \psi \phi(v)$ is not order conserving and that it has support in two blocks $P, Q$ of $A_{4}$ which are not in staircase form. Then there are multiplicity one summands $\eta_{1} \psi_{1} \phi_{1}$ and $\eta_{2} \psi_{2} \phi_{2}$ of $\eta \psi \phi$ 
(where $\eta_{1}, \eta_{2}$ are multiplicity one summands of $\eta ; \psi_{1}, \psi_{2}$ are multiplicity one summands of $\psi$; and $\phi_{1}, \phi_{2}$ are multiplicity one summands of $\phi)$ such that $\eta_{1} \psi_{1} \phi_{1}(v)$ meets $P$ and $\eta_{2} \psi_{2} \phi_{2}(v)$ meets $Q$. Thus there are rank one subordinates $v_{1}, v_{2}$ of $v$ such that $\eta_{1} \psi_{1} \phi_{1}\left(v_{1}\right)$ meets $P$ and $\eta_{2} \psi_{2} \phi_{2}\left(v_{2}\right)$ meets $Q$. It follows that the partial isometry $w=$ $\phi_{1}\left(v_{1}\right)+\phi_{2}\left(v_{2}\right)$ is not order conserving (since the map $\eta \psi$ is order conserving). However the argument above shows that $\eta \psi(w)$ is order conserving, which is the desired contradiction.

Remark. The proofs of Theorems 4.3 and 4.4 carry over readily to the case of systems of direct sums of finite dimensional nest algebras for which the embeddings have order preserving or order conserving partial embeddings between nest algebra summands.

Remark. In the terminology of Power [21] Theorem 4.4 establishes that the families of order preserving and order conserving embeddings are functorial families. It follows from Haworth and Power [9] that standard AF diagonal masas are unique up to approximately inner automorphism in the corresponding algebraic limit algebras. In particular the spectrum is a well defined and a complete invariant for these algebras.

\section{INVARIANTS AND CLASSIFICATIONS}

Let $A$ be a digraph algebra whose block diagonal subalgebra $A \cap A^{*}$ has minimal central projections $q_{1}, q_{2}, \ldots, q_{n}$. The reduced graph $H$ for $A$ is the graph with vertices labelled $1, \ldots, n$ and edges $(i, j)$ for which $q_{i} A q_{j}$ is non-zero. The abelian group $\mathbb{Z}^{\omega(A)}$, where $\omega(A)$ is the number of edges of $H$, may be defined intrinsically as the free abelian group $\mathcal{G}(A)$ whose generators are the equivalence classes $[v]$ of rank one elements $v$ of $\operatorname{Pisom}_{\text {reg }}(A)$, where the equivalence relation is the following: $v \sim w$ if, and only if, there are unitaries $u_{1}$ and $u_{2}$ in $A$ such that $v=u_{1} w u_{2}$. (Recall that $v \in \operatorname{Pisom}_{\text {reg }}(A)$ requires that $v^{*} v$ and $v v^{*}$ belong to $A$.) If $A$ is a finite dimensional nest algebra and $q_{1}, q_{2}, \ldots, q_{n}$ are the atoms of $A$, in order, then we write $T_{n}(\mathbb{Z})$ to denote the abelian group $\mathcal{G}(A)$. Note that the group homomorphisms $\pi_{f}: \mathcal{G}(A) \rightarrow K_{0}(A)$ and $\pi_{i}: \mathcal{G}(A) \rightarrow K_{0}(A)$ determined by the correspondences $[v] \mapsto\left[v v^{*}\right]$ and $[v] \mapsto\left[v^{*} v\right]$ respectively correspond to the row sum homomorphism and the column sum homomorphism from $T_{n}(\mathbb{Z})$ to $\mathbb{Z}^{n}$. Observe also that $K_{0}(A)$ can be identified in a natural way with a subgroup of $\mathcal{G}(A)$. For a full treatment of this invariant, see [20].

Definition 5.1. The dimension distribution group of the digraph algebra $A$ is the group $\mathcal{G}(A)$ described above. If $\mathcal{A}=\left\{A_{k}, \alpha_{k}\right\}$ is a regular 
star extendible system of digraph algebras, the dimension distribution group, $\mathcal{G}(\mathcal{A})$, of $\mathcal{A}$ is defined to be the direct limit $\underset{\lim }{\longrightarrow}\left(\mathcal{G}\left(A_{k}\right), \mathcal{G}\left(\alpha_{k}\right)\right)$ determined by the naturally induced embeddings.

Plainly, only the local regularity of $\alpha_{k}$ is necessary for the existence of the induced group homomorphisms $\mathcal{G}\left(\alpha_{k}\right)$. In view of the commuting diagram

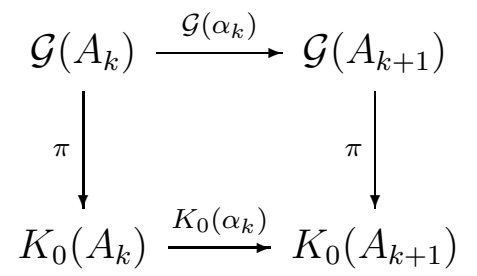

with $\pi=\pi_{i}$ or $\pi=\pi_{f}$, the invariant $\mathcal{G}(\mathcal{A})$ comes equipped with group homomorphisms $\pi_{i}: \mathcal{G}(\mathcal{A}) \rightarrow K_{0}(\mathcal{A})$ and $\pi_{f}: \mathcal{G}(\mathcal{A}) \rightarrow K_{0}(\mathcal{A})$ which are, in fact, scaled group homomorphisms with respect to the $K_{0}$ scale $\Sigma_{0}(\mathcal{A})$ and the dimension group scale $\Sigma(\mathcal{A}) \subseteq \mathcal{G}(\mathcal{A})$, which is determined by the regular partial isometries of the system $\mathcal{A}$. The natural full dimension distribution group invariant is therefore the quadruple

$$
\operatorname{Inv}(\mathcal{A})=\left((\mathcal{G}(\mathcal{A}), \Sigma(\mathcal{A})),\left(K_{0} \mathcal{A}, \Sigma_{0} \mathcal{A}\right), \pi_{f}, \pi_{i}\right)
$$

which is an invariant for regular star extendible isomorphism.

For self-adjoint systems, $\operatorname{Inv}(\mathcal{A})$ reduces to $\left(K_{0} \mathcal{A}, \Sigma_{0} \mathcal{A}\right)$. At the other extreme of triangular algebra systems (with regular embeddings), $\operatorname{Inv}(\mathcal{A})$ can be identified with a dual form of the fundamental binary relation (or spectrum) invariant. See [20]. In the discussion below we extend the scope for Inv as a classifying invariant.

In 20] it was shown that partly self-adjoint $T_{r}$-algebra systems are classified by Inv, together with an extra matrix unit scale, for $r=2,3$ but not for $r \geq 4$. Also, it was shown how one can introduce appropriate Grothendieck group invariants which capture the extra variety of regular embeddings and which serve as complete classifying invariants (at least for regular isomorphisms of systems). However, it is natural to enquire to what extent $\operatorname{Inv}(-)$ already serves as a basis for complete classification for systems of restricted type, such as order conserving embeddings. This is particularly so if it is known that the inner conjugacy class of an admissible embedding is determined by the induced map on $K_{0}(-)$ or on $\operatorname{Inv}(-)$.

5.1. Existence. Recall first the usual scheme for proving that an invariant such as $\operatorname{Inv}(-)$ is, in fact, a complete invariant for star extendible isomorphism. Start with an isomorphism $\Phi: \operatorname{Inv}(\mathcal{A}) \rightarrow \operatorname{Inv}\left(\mathcal{A}^{\prime}\right)$; 
this gives rise to a commuting diagram with crossover maps

$$
\operatorname{Inv}\left(A_{n_{1}}\right) \stackrel{\gamma_{1}}{\longrightarrow} \operatorname{Inv}\left(A_{m_{1}}^{\prime}\right) \stackrel{\delta_{1}}{\longrightarrow} \operatorname{Inv}\left(A_{n_{2}}\right) \longrightarrow \ldots
$$

Lift $\gamma_{1}$ to an admissible embedding $\phi_{1}: A_{n_{1}} \rightarrow A_{m_{1}}^{\prime}$, perhaps after increasing $m_{1}$. This step, the existence step, is usually the crux of the proof and some type of scale preservation is usually needed to enable the lifting. Similarly, lift $\delta_{1}$ to an admissible embedding $\psi_{1}$. Provided that admissible embeddings are closed under composition or that, for some other reason, the composition is admissible, appeal next to uniqueness to conclude that $\psi_{1} \circ \phi_{1}$ is conjugate to the given embedding $A_{n_{1}} \rightarrow A_{n_{2}}$. Adjust $\psi_{1}$ and obtain the commuting triangle for the first stage of the desired infinite commuting diagram.

We now obtain some existence results in the context of order conservation.

For a finite dimensional nest algebra $A$, write $\Sigma_{\text {oc }}(A)$ for the order conserving elements in the scale $\Sigma(A)$ of $\mathcal{G}(A)$. If $g$ lies in $\Sigma_{\mathrm{oc}}(A)$ then, under the identification of $\mathcal{G}(A)$ with $T_{r}(\mathbb{Z}), g$ is a non-negative integral matrix and has support in staircase form. Conversely, if $g$ has staircase form and $\pi_{i}(g) \in \Sigma_{0}(A)$ and $\pi_{f}(g) \in \Sigma_{0}(A)$ then $g \in \Sigma_{\text {oc }}(A)$. In a similar way, define $\Sigma_{\text {op }}(A)$, the subset of order preserving elements.

We are interested in lifting a $\pi$-respecting group homomorphism $\gamma: \mathcal{G}\left(A_{1}\right) \rightarrow \mathcal{G}\left(A_{2}\right)$ to a star extendible regular embedding. The possible order conservation and order preservation properties which we might require of $\gamma$ are that it be locally order conserving, locally order preserving, order conserving, or order preserving. (Order conservation or order preservation for $\gamma$ means, of course, that $\gamma$ maps $\Sigma_{\text {oc }}$ into $\Sigma_{\text {oc }}$ or $\Sigma_{\text {op }}$ into $\Sigma_{\text {op }}$, respectively. The local versions mean that $\gamma$ maps elements of $\Sigma_{\mathrm{oc}}$ which have only one non-zero entry into $\Sigma_{\mathrm{oc}}$ or $\Sigma_{\mathrm{op}}$, as appropriate.) Of course, if $\gamma$ can be lifted to a regular embedding $\phi$ with $\mathcal{G}(\phi)=\gamma$, then $\phi$ will necessarily have the same order conservation and order preservation properties as $\gamma$. Since an embedding of any of these types is necessarily locally order conserving, it follows from Theorem 5.2 that locally order preserving embeddings, order preserving embeddings, and order conserving embeddings all have liftings of the corresponding type.

Theorem 5.2. Let $A_{1}$ and $A_{2}$ be finite dimensional nest algebras. Let $\gamma: \mathcal{G}\left(A_{1}\right) \rightarrow \mathcal{G}\left(A_{2}\right)$ be a locally order conserving homomorphism which is $\pi$-respecting and for which the restriction of $\gamma$ to $K_{0}\left(A_{1}\right)$ is a scaled group homomorphism. Then there is a locally order conserving star extendible embedding $\phi: A_{1} \rightarrow A_{2}$ for which $\mathcal{G}(\phi)=\gamma$. 
While the proof of the theorem is somewhat technical, the existence of liftings of locally order preserving embeddings is quite elementary for the following reason. With the obvious notation, note that one can lift the images $\gamma\left(\left[e_{12}\right]\right), \gamma\left(\left[e_{23}\right]\right), \ldots, \gamma\left(\left[e_{r-1, r}\right]\right)$ to order preserving regular partial isometries $v_{1}, v_{2}, \ldots, v_{r-1}$ in $A_{2}$ with matching initial and final projections

$$
v_{1}^{*} v_{1}=v_{2} v_{2}^{*}, v_{2}^{*} v_{2}=v_{3} v_{3}^{*}, \ldots, v_{r-2}^{*} v_{r-2}=v_{r-1} v_{r-1}^{*} .
$$

(This uses the fact that $\gamma$ preserves the scale and respects the group homomorphisms $\pi_{f}$ and $\pi_{i}$.) Since the order preserving partial isometries form a semigroupoid (admissible products of order preserving partial isometries are order preserving) we can define $\phi$ as the unique star extendible embedding for which $\phi\left(e_{i, i+1}\right)=v_{i}, 1 \leq i \leq r-1$. Now $\gamma$ and $G(\phi)$ are locally order preserving homomorphisms which are $\pi$ respecting and agree on $\left[e_{11}\right], \ldots,\left[e_{r r}\right]$. Since $\pi_{i}$ and $\pi_{f}$ determine an order preserving element it follows that $\gamma=G(\phi)$.

This approach is not available for locally order conserving embeddings (since a product of two order conserving partial isometries can be a partial isometry which is not order conserving). However, Theorem 5.2 follows quickly from the following lemma. To clarify the formulation of the lemma we remark that it is used to lift $\gamma\left(\left[e_{12}\right]\right)$ to a partial isometry which has order conserving products with a priori liftings of $\gamma\left(\left[e_{i j}\right]\right)$, for $i \geq 2$. This in turn enables an immediate proof of Theorem 5.2 by induction.

Lemma 5.3. Let $X=\left(x_{i j}\right), Y^{k}=\left(y_{i j}^{k}\right)$, and $Z^{k}=\left(z_{i j}^{k}\right), 1 \leq k \leq t$, be matrices with non-negative integral entries and with order conserving staircase form. Suppose that $X$ is $p \times q, Y^{k}$ is $q \times r_{k}, Z^{k}$ is $p \times r_{k}$ and that $\pi_{f}(X)=\pi_{f}\left(Z^{k}\right), \pi_{i}(X)=\pi_{f}\left(Y^{k}\right)$, and $\pi_{i}\left(Y^{k}\right)=\pi_{i}\left(Z^{k}\right)$, for $1 \leq k \leq t$. Suppose moreover that $v_{1}, \ldots, v_{t}$ are block matrices which are regular partial isometries, with common final projections, for which each $v_{k}$ has rank distribution $Y^{k}$. Then there is a regular partial isometry $u$, with rank distribution $X$ and initial projection equal to $v_{1} v_{1}^{*}$, for which the products $u v_{k}$ are regular partial isometries with rank distribution $Z^{K}$, for $1 \leq k \leq t$.

Proof. It will be enough to show that the assertion of the lemma follows if it is assumed that the lemma is true in the case of matrices of sizes $p^{\prime} \times q^{\prime}, q^{\prime} \times r_{k}^{\prime}$ and $p^{\prime} \times r_{k}^{\prime}$, where $p^{\prime} \leq p, q^{\prime} \leq q, r_{k}^{\prime} \leq r_{k}, 1 \leq k \leq t$, and at least one of these inequalities is strict.

We may assume that all the entries $x_{11}, y_{11}^{k}$ and $z_{11}^{k}, 1 \leq k \leq t$ are non-zero. The reason for this is that if a staircase form matrix has its first entry equal to zero, then either the first row or the first column 
of the matrix is zero; the assertion then follows from the induction hypothesis.

Let $d=\min \left\{x_{11}, y_{11}^{k}, z_{11}^{k} \mid 1 \leq k \leq t\right\}$. Let $e$ be a subprojection of $v_{1} v_{1}^{*}\left(=v_{i} v_{i}^{*}\right.$ for all $\left.i\right)$ which has rank $d$; let $v_{k}^{\prime}=e v_{k}$; and let $u^{\prime}$ be a regular partial isometry of rank $d$ with initial projection $e$ and with $p \times q$ rank distribution matrix of the form:

$$
\left[\begin{array}{cccc}
d & 0 & \ldots & 0 \\
0 & 0 & \ldots & 0 \\
\vdots & \vdots & & \vdots \\
0 & 0 & \ldots & 0
\end{array}\right]
$$

Set $z_{k}^{\prime}=u^{\prime} v_{i}^{\prime}\left(=u^{\prime} v_{i}\right)$, so that $z_{k}^{\prime}$ has a similar rank distribution matrix, of size $p \times r_{k}$. Consider now the matrices $\hat{X}, \hat{Y}_{k}$, and $\hat{Z}_{k}$, which are obtained from $X, Y_{k}$, and $Z_{k}$ by subtracting $d$ form the first entry, together with the partial isometries $\hat{v}_{k}=v_{k}-v_{k}^{\prime}$ and notice that these satisfy the hypotheses of the lemma. By the induction hypothesis, we may assume that there is a lifting $\hat{u}$ for $\hat{X}$ with $\hat{u}^{*} \hat{u}=\hat{v}_{k} \hat{v}_{k}^{*}$, for all $k$, such that $\hat{u} \hat{v}_{k}$ has rank distribution $\hat{Z}_{k}$. Now $u=u^{\prime}+\hat{u}$ is a partial isometry with the properties needed to prove the lemma.

5.2. Classifications. We are now in a position to obtain a variety of classification results. Let $\mathcal{F}_{\text {loc }}$ be the family of embeddings between finite dimensional nest algebras which are locally order conserving and let Sys $\left(\mathcal{F}_{\text {loc }}\right)$ be the family of systems $\mathcal{A}$ for which the given embeddings and their compositions are locally order conserving. Similarly define the families $\operatorname{Sys}\left(\mathcal{F}_{\text {oc }}\right)$, Sys $\left(\mathcal{F}_{\text {lop }}\right)$ and $\operatorname{Sys}\left(\mathcal{F}_{\text {op }}\right)$ for order conserving, locally order preserving and order preserving embeddings.

Recall that an isomorphism $\gamma: \operatorname{Inv}(\mathcal{A}) \longrightarrow \operatorname{Inv}\left(\mathcal{A}^{\prime}\right)$ is an isomorphism of $\mathcal{G}(\mathcal{A})$ onto $\mathcal{G}\left(\mathcal{A}^{\prime}\right)$ which respects $K_{0}$, the scales, $\pi_{i}$, and $\pi_{f}$. More specifically, $\gamma: \mathcal{G}(\mathcal{A}) \longrightarrow \mathcal{G}\left(\mathcal{A}^{\prime}\right)$ satisfies:

$$
\begin{aligned}
\gamma(\Sigma(\mathcal{A})) & =\Sigma\left(\mathcal{A}^{\prime}\right), \\
\gamma\left(K_{0}(\mathcal{A})\right) & =K_{0}\left(\mathcal{A}^{\prime}\right), \\
\gamma\left(\Sigma_{0}(\mathcal{A})\right) & =\Sigma_{0}\left(\mathcal{A}^{\prime}\right), \\
\gamma \circ \pi_{i} & =\pi_{i} \circ \gamma, \\
\gamma \circ \pi_{f} & =\pi_{f} \circ \gamma .
\end{aligned}
$$

In addition to the scale $\Sigma(\mathcal{A})$ in $G(\mathcal{A})$ we define the following subscales. 
Definition 5.4. The scale $\Sigma_{\text {oc }}(\mathcal{A})$ (resp. $\Sigma_{\text {op }}(\mathcal{A})$ ) consists of the images of the classes $[v]$ in $G\left(A_{k}\right)$ for which $[i(v)]$ belongs to $\Sigma_{\text {oc }}\left(A_{n}\right)$ (resp. $\left.\Sigma_{\mathrm{op}}\left(A_{n}\right)\right)$ for all the system maps $i: A_{k} \rightarrow A_{n}$, for $n>k$.

We now define order conservation and local order conservation for scale preserving homomorphisms between the dimension distribution groups of systems. This is, of course, based on the corresponding definitions in the context of dimension distribution groups of digraph algebras (see the paragraph preceding Theorem 5.2). One can verify that $\gamma$ is order conserving if, and only if, $\gamma\left(\Sigma_{\text {oc }}(\mathcal{A})\right) \subseteq \Sigma_{\text {oc }}\left(\mathcal{A}^{\prime}\right)$. The order preserving and locally order preserving homomorphisms are defined similarly.

Definition 5.5. Let $\gamma:(\mathcal{G}(\mathcal{A}), \Sigma(\mathcal{A})) \longrightarrow\left(\mathcal{G}\left(\mathcal{A}^{\prime}\right), \Sigma\left(\mathcal{A}^{\prime}\right)\right)$ be a scale preserving homomorphism of dimension distribution groups so that for each $n$, there is an integer $k_{n}$ such that $\gamma\left(\mathcal{G}\left(A_{n}\right)\right) \subseteq \mathcal{G}\left(A_{k_{n}}^{\prime}\right)$. We say that $\gamma$ is order conserving if, for each $n$, the restriction of $\gamma$ to $\mathcal{G}\left(A_{n}\right)$ is order conserving as a map from $\mathcal{G}\left(A_{n}\right)$ into $\mathcal{G}\left(A_{k_{n}}^{\prime}\right)$. Similarly, $\gamma$ is locally order conserving if each such restriction is locally order conserving.

The following theorem gives in particular a sufficient condition for the regular isomorphism of systems in which all compositions are locally order conserving.

Theorem 5.6. Let $\mathcal{A}$ and $\mathcal{A}^{\prime}$ be systems of finite dimensional nest algebras such that all compositions of embeddings are locally order conserving. Then the following statements are equivalent.

1. $\mathcal{A}$ and $\mathcal{A}^{\prime}$ are isomorphic with locally order conserving crossover maps.

2. $\operatorname{Inv}(\mathcal{A})$ and $\operatorname{Inv}\left(\mathcal{A}^{\prime}\right)$ are isomorphic via a locally order conserving isomorphism.

Proof. The usual scheme of proof that 2. implies 1. can be completed as follows. With the aid of Theorem 5.2, obtain a lifting of a suitable restriction

$$
\gamma_{1}:\left(\mathcal{G}\left(A_{n_{1}}\right), \Sigma\left(A_{n_{1}}\right)\right) \rightarrow\left(\mathcal{G}\left(A_{m_{1}}^{\prime}\right), \Sigma\left(A_{m_{1}}^{\prime}\right)\right)
$$

to a locally order conserving star extendible homomorphism $\phi_{1}$. In the same way, the restriction of $\gamma^{-1}$ to $\mathcal{G}\left(A_{m_{1}}^{\prime}\right)$ may be lifted to a locally order conserving embedding $\psi_{1}$. Since $\mathcal{G}\left(\psi_{1} \circ \phi_{1}\right)=\mathcal{G}(\beta)$, where $\beta$ is a composition of the given embeddings for $\mathcal{A}$, it folows that $\psi_{1} \circ \phi_{1}$ is actually locally order conserving. From Theorem 3.2 we can replace $\psi_{1}$ by an inner unitary conjugate to obtain $\psi_{1} \circ \phi_{1}=\beta$. Continuing in this way, we obtain a commuting diagram for the desired isomorphism. 
The same proof also works in the order conserving case; the next theorem classifies order conserving systems of finite dimensional nest algebras up to order conserving commuting diagram isomorphism.

Theorem 5.7. Let $\mathcal{A}$ and $\mathcal{A}^{\prime}$ be systems of finite dimensional nest algebras with order conserving embeddings. Then the following statements are equivalent.

1. $\mathcal{A}$ and $\mathcal{A}^{\prime}$ are isomorphic with order conserving crossover maps.

2. $\operatorname{Inv}(\mathcal{A})$ and $\operatorname{Inv}\left(\mathcal{A}^{\prime}\right)$ are isomorphic via an order conserving isomorphism.

We also have parallel theorems for the order preserving and locally order preserving cases. The proofs are simpler in that Theorem 5.2 may be replaced by the simpler lifting argument above for locally order preserving homomorphisms.

Theorem 5.8. Let $\mathcal{A}$ and $\mathcal{A}^{\prime}$ be systems of finite dimensional nest algebras with order preserving embeddings. Then the following statements are equivalent.

1. $\mathcal{A}$ and $\mathcal{A}^{\prime}$ are isomorphic with order preserving crossover maps.

2. $\operatorname{Inv}(\mathcal{A})$ and $\operatorname{Inv}\left(\mathcal{A}^{\prime}\right)$ are isomorphic via an order preserving isomorphism.

Theorem 5.9. Let $\mathcal{A}$ and $\mathcal{A}^{\prime}$ be systems of finite dimensional nest algebras such that all compositions of embeddings are locally order preserving. Then the following statements are equivalent.

1. $\mathcal{A}$ and $\mathcal{A}^{\prime}$ are isomorphic with locally order preserving crossover maps.

2. $\operatorname{Inv}(\mathcal{A})$ and $\operatorname{Inv}\left(\mathcal{A}^{\prime}\right)$ are isomorphic via a locally order preserving isomorphism.

The theorems above and also Theorem 5.10 have exact counterparts for limits of direct sums of finite dimensional nest algebras

Remark. It is natural to ask to what extent the hypotheses of the theorems above can be relaxed. It can be shown for example that if $\theta: K_{0}\left(A_{1}\right) \rightarrow K_{0}\left(A_{2}\right)$ is an isomorphism preserving the order preserving algebraic order $S_{\mathrm{op}}(-)$ then there is a unique (up to conjugacy) locally order preserving lifting $\phi: A_{1} \rightarrow A_{2}$. Here, $S_{\text {op }}(A)$ is the set of pairs $\left(\left[v v^{*}\right],\left[v^{*} v\right]\right)$ in $\Sigma_{0}(A) \times \Sigma_{0}(A)$ arising from order preserving partial isometries.) This suggests that at the level of systems, an isomorphism $\gamma: K_{0}(\mathcal{A}) \rightarrow K_{0}\left(\mathcal{A}^{\prime}\right)$ which maps $S_{\text {op }}\left(\mathcal{A}_{1}\right)$ to $S_{\text {op }}\left(\mathcal{A}_{2}\right)$ may lift to an isomorphism of systems. On the other hand, a composition of locally order preserving embeddings is not necessarily locally order 
preserving and so the usual proof is not available. (See also Power 18 , errata].) This suggests that such an isomorphism may not be sufficient for system or algebra isomorphism. In fact this problem is already present in the case of triangular systems where the order $S_{\text {op }}(-)$ agrees with the algebraic order $S(-)$. One way to settle the issue would be to show that even for alternation algebras the invariant $\left(K_{0}(-), S(-)\right)$ is not a complete invariant.

Next we give applications of Theorem 5.7 and 5.8 to the classification of algebraic limits of order conserving and order preserving systems.

Let $\mathcal{A}$ belong to $\operatorname{Sys}\left(\mathcal{F}_{\text {oc }}\right)$ or to $\operatorname{Sys}\left(\mathcal{F}_{\text {op }}\right)$ with algebraic direct limit $A_{0}=\underset{\operatorname{alg} \lim }{\mathcal{A}}$. It follows from Theorem 4.3 that we may define $\operatorname{Inv}\left(A_{0}\right)=\operatorname{Inv}(\mathcal{A})$ as an invariant for star extendible isomorphism. The next theorem shows that, in the Sys $\left(\mathcal{F}_{\text {oc }}\right)$ case, the definition $\Sigma_{\mathrm{oc}}\left(A_{0}\right)=\Sigma_{\mathrm{oc}}(\mathcal{A})$ also gives an invariant and that $\left(\operatorname{Inv}\left(A_{0}\right), \Sigma_{\mathrm{oc}}\left(A_{0}\right)\right)$ is a complete invariant.

Theorem 5.10. Let $\mathcal{A}, \mathcal{A}^{\prime}$ be order conserving systems of finite dimensional nest algebras and let $A_{0}, A_{0}^{\prime}$ be their algebraic direct limits. Then $A_{0}$ and $A_{0}^{\prime}$ are star extendibly isomorphic if, and only if, there is an isomorphism $\gamma: \operatorname{Inv}\left(A_{0}\right) \rightarrow \operatorname{Inv}\left(A_{0}^{\prime}\right)$ such that $\gamma\left(\Sigma_{o c}\left(A_{0}\right)\right)=\Sigma_{o c}\left(A_{0}^{\prime}\right)$

Proof. In view of Theorem 5.7, it will be sufficient to show that if $A_{0}, A_{0}^{\prime}$ are star extendibly isomorphic then not only is the induced isomorphism of $\mathcal{A}, \mathcal{A}^{\prime}$ regular, but the commuting diagram isomorphism may be implemented by order conserving embeddings. However this follows immediately from Lemma 4.5

And, of course, there is an analogous theorem in the order preserving context, with $\Sigma_{\mathrm{op}}\left(A_{0}\right)=\Sigma_{\mathrm{op}}(\mathcal{A})$ an invariant and $\left(\operatorname{Inv}\left(A_{0}\right), \Sigma_{\mathrm{op}}\left(A_{0}\right)\right)$ a complete invariant.

Theorem 5.11. Let $\mathcal{A}, \mathcal{A}^{\prime}$ be order preserving systems of finite dimensional nest algebras and let $A_{0}, A_{0}^{\prime}$ be their algebraic direct limits. Then $A_{0}$ and $A_{0}^{\prime}$ are star extendibly isomorphic if, and only if, there is an isomorphism $\gamma: \operatorname{Inv}\left(A_{0}\right) \rightarrow \operatorname{Inv}\left(A_{0}^{\prime}\right)$ such that $\gamma\left(\Sigma_{o p}\left(A_{0}\right)\right)=\Sigma_{o p}\left(A_{0}^{\prime}\right)$

It is a consequence of Theorem 3.6 of Donsig [2] that the operator algebra limits of order preserving systems of finite dimensional nest algebras are star extendibly isomorphic if, and only if, the algebraic limit algebras are isomorphic. Combining this with Theorem 5.10 it follows that the operator algebras of order preserving systems may be classified by $\left(\operatorname{Inv}(-), \Sigma_{\mathrm{oc}}(-)\right)$. We anticipate that the same is true in the order conserving case.

We conclude with some examples. 
Example 1. Let $A_{k}=T_{2^{k}} \otimes M_{2}$ and consider the embeddings $\phi_{k}: A_{k} \longrightarrow$ $A_{k+1}$ given by $\phi(a)=a \oplus a$ (standard type embeddings). Then $A=$ $\lim _{\longrightarrow}\left(A_{k}, \phi_{k}\right) \cong S \otimes M_{2}$, where $S=\lim _{\longrightarrow}\left(T_{2^{k}}, \sigma_{k}\right)$ is the standard upper triangular $2^{\infty}$ limit algebra. Let $X_{0}=\prod_{i=1}^{\infty}\{0,1\}$ with the product topology. The spectrum (groupoid) $R\left(C^{*}(S)\right)$ is the 'tails the same' equivalence relation on $X_{0}$ and the spectrum $R(S)$ is the reverse lexicographic sub-relation. $R\left(M_{2}\right)=\{0,1\} \times\{0,1\}$, the full equivalence relation on $\{0,1\} . R(A)=R\left(M_{2}\right) \times R(S)$, the product relation acting on $X=\{0,1\} \times X_{0}$. We have

(i) $K_{0}(A) \cong C\left(X_{0}, \mathbb{Z}\right)$, the continuous integer valued functions on $X_{0}$.

(ii) $\Sigma_{0}(A)=\left\{\alpha \in K_{0}(A) \mid 0 \leq \alpha(x) \leq 2\right.$, for all $\left.x \in X_{0}\right\}$.

(iii) $\mathcal{G}(A) \cong C_{c}(R(S), \mathbb{Z})$, the continuous integer valued functions on $R(S)$ with compact support.

We may make the following interpretation: for each $p \in R(S)$, let $K_{0}(p)=K_{0}\left(M_{2}\right)$; then $\mathcal{G}(A)$ can be identified with the space of compactly supported continuous functions on $R(S)$ such that $\phi(p) \in K_{0}(p)$, for all $p$.

Example 2. Let $B_{0}$ be the unital algebraic limit algebra $B_{0}=\underline{\operatorname{alg} \lim }\left(T_{n_{k}}, \phi_{k}\right)$ where the embeddings $\phi_{k}$ are order preserving. Let

$$
A_{0}=\underline{\operatorname{alg} \lim }\left(T_{n_{k}} \otimes M_{m_{k}}, \phi_{k} \otimes \psi_{k}\right)
$$

where each embedding $\psi_{k}: M_{m_{k}} \rightarrow M_{m_{k+1}}$ is a unital $\mathrm{C}^{*}$-algebra injection. Then $A_{0}=B_{0} \otimes D_{0}$ with $D_{0}$ a unital ultramatricial algebra. As we observed at the end of Section 3, the maps $\phi_{k} \otimes \psi_{k}$ and their compositions are order preserving. In the degenerate case $\phi_{k}=i d: T_{2} \rightarrow T_{2}$, $\mathcal{G}\left(A_{0}\right)$ is naturally isomorphic to $T_{2}(\mathbb{Z}) \otimes_{\mathbb{Z}} K_{0}\left(D_{0}\right)$. More generally $\mathcal{G}\left(A_{0}\right)$ can be identified naturally in terms of $K_{0}\left(D_{0}\right)$-valued functions on the spectrum (semigroupoid) $R\left(B_{0}\right)$ of $B_{0}$.

Example 3. Let $B_{0}$ be the unital locally finite algebra $B_{0}=\underline{\operatorname{alg} \lim }\left(M_{n_{k}}, \sigma_{k}\right)$ where the embeddings $\sigma_{k}$ are standard embeddings (of direct sum form) with respect to the usual matrix unit systems. Let $A_{k} \subseteq M_{n_{k}}$ be a nest algebra whose nest projections are ordered in a compatible way with the diagonal matrix units and suppose that $\sigma_{k}\left(A_{k}\right) \subseteq A_{k+1}$ for all $k$. Since the multiplicity one decomposition of $\sigma_{k}$ is necessarily an ordered sum, it is clear that each $\sigma_{k}$ is order conserving as a map from $A_{k}$ to $A_{k+1}$. Plainly the algebra $A_{0}$ contains the triangular algebra $T_{0}^{\sigma}=\underset{\operatorname{alg} \lim }{\longrightarrow}\left(T_{n_{k}}, \sigma_{k}\right)$. In fact it is the case that every intermediate algebra $A_{0}^{\prime}$, with the property $T_{0}^{\sigma} \subseteq A_{0}^{\prime} \subseteq B_{0}$ is necessarily of this 
form. This can be seen by a straightforward application of inductivity arguments (Chapter 4 of [18]) in the setting of algebraic direct limits; $A_{0}^{\prime}=\underset{\operatorname{alg} \lim }{\longrightarrow}\left(A_{k}, \sigma_{k}\right)$ where $A_{k}^{\prime}=A_{0} \cap C^{*}\left(A_{k}\right)$, and since $A_{k}^{\prime}$ contains $T_{n_{k}}$, for each $k, A_{k}^{\prime}$ is a finite dimensional nest algebra. Also it follows similarly that if $A^{\prime}$ is a closed subalgebra of a UHF $\mathrm{C}^{*}$-algebra which contains a triangular standard embedding limit algebra, then $A^{\prime}$ is an order conserving limit of finite dimensional nest algebras.

Example 4. In analogy with the last example consider a refinement limit presentation of $B_{0}$ together with its associated triangular refinement limit algebra $T_{0}^{\rho}$. Then it can be shown that every intermediate algebra is an order conserving refinement limit of finite dimensional nest algebras. This verification depends on the inductivity of the intermediate algebras and the fact that a refinement type embedding between finite dimensional nest algebras is order conserving.

The most transparent intermediate algebras in this case are those nest subalgebras of $B_{0}$ determined by a finite nest of $T_{0}^{\rho}$-invariant projections. More generally however the intermediate algebras are nest subalgebras of $B_{0}$ determined by a nest of $T_{0}^{\rho}$-invariant projections in the weak closure of $B_{0}$ in the tracial representation.

Example 5. In a similar way one obtains that an intermediate operator algebra $A$ satisfying $B \subseteq A \subseteq C^{*}(B)$, where $B$ is an alternation algebra, is an order conserving limit of finite dimensional nest algebras. In fact, the same result is valid whenever $A$ is an intermediate algebra between a direct limit of $T_{n}$ 's with order preserving embedding and its enveloping $\mathrm{C}^{*}$-algebra.

\section{REFERENCES}

[1] R. L. Baker, Triangular UHF algebras, J. Funct. Anal. 91 (1990), 182-212.

[2] A. P. Donsig, Algebraic orders and chordal limit algebras, Proc. Edinburgh Math. Soc. 41 (1998), 465-485.

[3] A. P. Donsig and A. Hopenwasser, Order preservation in limit algebras, J. Functional Analysis 133 (1995), 342-394.

[4] A. P. Donsig and S. C. Power, The failure of approximate inner equivalence of standard diagonals in regular limits of digraph algebras, Bull. Canadian Math. Soc. 39 (1996), 420-428.

[5] - Homology for operator algebras IV, J. Functional Analysis 150 (1997), $240-287$.

[6] - The classification of limits of 2 -cycle algebras, Indiana Univ. Math. J. 48 (1999), 411-127.

[7] G. A. Elliott, On the classification of inductive limits of sequences of semisimple finite-dimensional algebras, J. Algebra 38 (1976), 29-44. 
[8] J. Glimm, On a certain class of operator algebras, Trans. Amer. Math. Soc. 95 (1960), 318-340.

[9] P.A. Haworth and S.C. Power, On the uniqueness of AF diagonals in regular limit algebras, preprint, Lancaster University, Nov. 2000.

[10] D. Hefferman, Uniformly $T_{2}$ algebras in approximately finite-dimensional $C^{*}$ algebras, J. London Math. Soc. 55 (1997), 181-192.

[11] A. Hopenwasser and S. C. Power, Classification of limits of upper triangular matrix algebras, Proc. Edinburgh Math. Soc. 36 (1992), 107-121.

[12] P. S. Muhly and B. Solel, Subalgebras of groupoid $C^{*}$-algebras, J. Reine Angew. Math. 402 (1989), 41-75.

[13] J. R. Peters, Y.-T. Poon, and B. H. Wagner, Triangular AF algebras, J. Operator Theory 23 (1990), 81-114.

[14] Y.-T. Poon, A complete isomorphism invariant for a class of triangular UHF algebras, J. Operator Theory 27 (1992), 221-230.

[15] S. C. Power, Classification of tensor products of triangular operator algebras, Proc. London Math. Soc. 61 (1990), 571-614.

[16] - The classification of triangular subalgebras of $A F C^{*}$-algebras, Bull. London Math. Soc. 22 (1990), 269-272.

[17] S. C. Power, Algebraic orders on $K_{0}$ and approximately finite operator algebras, J. Operator Theory 27 (1992), 87-106.

[18] S. C. Power, Limit algebras: An introduction to subalgebras of $C^{*}$-algebras, Pitman Research Notes in Mathematics Series, vol. 278, Longman Scientific and Technical, England, New York, 1992, Errata available at www.maths.lancs.ac.uk/ ${ }^{\sim}$ power/pubs.brief.html.

[19] _ Relative positions of matroid algebras, J. Funct. Anal 165 (1999), $205-239$.

[20] — Grothendieck group invariants for partly self-adjoint operator algebra, Intern. J. Math. 11 (2000), 41-64.

[21] _ _ Approximately finitely acting operator algebras, preprint, 2000.

Dept. of Mathematics, University of Alabama, Tuscaloosa, AL 35487, U.S.A.

E-mail address, Alan Hopenwasser: ahopenwa@euler.math.ua.edu

Dept. of Mathematics \& Statistics, Lancaster University, LanCASTER, U.K. LA1 4YF

E-mail address, Stephen C. Power: s.power@lancaster.ac.uk 